\title{
Statin-boosted cellular uptake and endosomal escape of penetratin due to reduced membrane dipole potential
}

\author{
Gyula Batta $^{1,2}$ (1) | Levente Kárpáti ${ }^{3,4}$ | Gabriela Fulaneto Henrique ${ }^{2}$ | \\ Gabriella Tóth $^{1}$ | Szabolcs Tarapcsák ${ }^{1,5}$ | Tamas Kovacs ${ }^{1}$ | Florina Zakany ${ }^{1}$ | \\ István M. Mándity ${ }^{3,4}$ () | Peter Nagy ${ }^{1}$ (i) \\ ${ }^{1}$ Department of Biophysics and Cell Biology, Faculty of Medicine, University of Debrecen, Debrecen, Hungary \\ ${ }^{2}$ Department of Genetics and Applied Microbiology, Faculty of Science and Technology, University of Debrecen, Debrecen, Hungary \\ ${ }^{3}$ Department of Organic Chemistry, Faculty of Pharmacy, Semmelweis University, Budapest, Hungary \\ ${ }^{4}$ TTK Lendület Artificial Transporter Research Group, Institute of Materials and Environmental Chemistry, Research Center for Natural Sciences, Budapest, Hungary \\ ${ }^{5}$ Utah Center for Genetic Discovery, Eccles Institute of Human Genetics, University of Utah, Salt Lake City, Utah, USA
}

\section{Correspondence}

Peter Nagy, Department of Biophysics and Cell Biology, Faculty of Medicine, University of Debrecen, Debrecen, Hungary.

Email: nagyp@med.unideb.hu

István M. Mándity, TTK Lendület Artificial Transporter Research Group, Institute of

Materials and Environmental Chemistry,

Research Center for Natural Sciences,

Budapest, Hungary

Email: mandity.istvan@ttk.hu

Funding information

Magyar Tudományos Akadémia; Nemzeti Kutatási, Fejlesztési és Innovációs Hivatal, Grant/Award Numbers: 2018-1.2.1-NKP, GINOP-2.3.2-15-2016-00020, GINOP2.3.2-15-2016-00044, K120302, ANN133421
Background and Purpose: Cell penetrating peptides are promising tools for delivery of cargo into cells, but factors limiting or facilitating their cellular uptake are largely unknown. We set out to study the effect of the biophysical properties of the cell membrane on the uptake of penetratin, a cell penetrating peptide.

Experimental Approach: Using labelling with $\mathrm{pH}$-insensitive and $\mathrm{pH}$-sensitive dyes, the kinetics of cellular uptake and endo-lysosomal escape of penetratin were studied by flow cytometry.

Key Results: We report that escape of penetratin from acidic endo-lysosomal compartments is retarded compared with its total cellular uptake. The membrane dipole potential, known to alter transmembrane transport of charged molecules, is shown to be negatively correlated with the concentration of penetratin in the cytoplasmic compartment. Treatment of cells with therapeutically relevant concentrations of atorvastatin, an inhibitor of HMG-CoA reductase and cholesterol synthesis, significantly increased endosomal escape of penetratin in two different cell types. This effect of atorvastatin correlated with its ability to decrease the membrane dipole potential.

Conclusion and Implications: These results highlight the importance of the dipole potential in regulating cellular uptake of cell penetrating peptides and suggest a clinically relevant way of boosting this process.

KEYWORDS

atorvastatin, cell penetrating peptide, dipole potential, lysosomal escape, penetratin 


\section{1 | INTRODUCTION}

During the past decades, there has been growing interest in cell penetrating peptides (CPPs) that can traverse biological membranes. Cell penetrating peptides are a diverse set of short peptide sequences that usually consist of 30 or fewer amino acids and can be classified as either cationic, amphipathic or hydrophobic. Cell penetrating peptides are important because of their ability to cross cell membranes in a nontoxic manner and because of their capacity to support efficient delivery of cell-impermeable therapeutic cargos with MWs several times greater than their own (Guidotti et al., 2017). Several natural peptides with cell penetration capability have been characterized including substance P analogues, the Tat protein in HIV and the homeodomain of the Antennapedia protein in Drosophila (Joliot et al., 1991; Repke \& Bienert, 1987). The cell translocation sequence was localized to the third helix of the homeodomain leading to the development of a 16-amino acid oligopeptide rich in positively charged amino acids. This peptide, penetratin, belongs to the cationic class of cell penetrating peptides and is widely used in research aimed at defining the mechanisms of cellular uptake of peptides (Derossi et al., 1994).

Although cell penetrating peptides hold great promise in drug delivery, their clinical potential is currently limited by low bioavailability, short half-life and lack of specificity (Fominaya et al., 2015; Qian et al., 2016; Wang et al., 2014). This latter shortcoming can be remedied by equipping the cell penetrating peptide with a homing domain or by fusing it to an inhibitory domain made up of negatively charged residues that is removed in the tumour micro-environment having increased proteolytic activity (Jiang et al., 2004; Wang et al., 2014). The mechanism of cellular entry of cell penetrating peptides also limits their efficiency putting it at the forefront of current investigations. One of the two, well-established routes of cellular entry for cell penetrating peptides is direct plasma membrane translocation, which may involve formation of inverted micelles, transient pores or increased fluidity of the plasma membrane (Guidotti et al., 2017; Ziegler, 2008). Another well-established route of cellular entry for cell penetrating peptides is endocytosis (Futaki, 2006; Rezgui et al., 2016). Unless the endocytic uptake itself is followed by endosomal escape, the cell penetrating peptide does not gain access to the cytosolic compartment and is digested in lysosomes. Many studies focused on the release of cell penetrating peptides from endosomes, leading to the insertion of endosomolytic sequences into or covalent coupling of endosomolytic compounds to cell penetrating peptides (Erazo-Oliveras et al., 2012; Nakase et al., 2011).

Numerous other approaches have been adopted to increase the cellular uptake of cell penetrating peptides including backbone cyclization, unnatural amino acids, pegylation and acylation (Erazo-Oliveras et al., 2012; Lönn et al., 2016; Najjar et al., 2017; Wallbrecher et al., 2014). The problem is further complicated by the fact that cellular uptake in 3D tumour spheroids is not strongly correlated with the uptake in monolayers (van den Brand et al., 2018). Other strategies for improving cellular delivery are based on the realization that a

\section{What is already known}

- Cell penetrating peptides traverse intact membranes even when loaded with cargo.

- The effect of membrane biophysical properties on the uptake process is unclear in live cells.

\section{What does this study add}

- The positive, intramembrane dipole potential inhibits uptake and endo-lysosomal escape of penetratin in live cells.

- Atorvastatin treatment decreases the membrane dipole potential and also enhances the cellular uptake of penetratin.

\section{What is the clinical significance}

- Decreasing membrane dipole potential can boost the uptake of drugs coupled to cell penetrating peptides.

cell penetrating peptide must cross a membrane independent of its uptake mechanism. The direct translocation mechanism involves crossing the plasma membrane, whereas the endocytic mechanism relies on traversing membranes of the endolysosomal compartment. Due to their charged nature, electrostatic interactions of cell penetrating peptides with anionic phospholipids and heparan sulphate proteoglycans have been implicated in direct membrane translocation and endocytosis, respectively (Poon \& Gariepy, 2007; Thoren et al., 2004). Transport of charged substances across the plasma membrane is also influenced by the three different kinds of membrane potentials, the transmembrane, the surface and the dipole potential (O'Shea, 2003). The magnitude of the dipole potential, generated by the preferential orientation of lipids and interfacial water molecules, is approximately 200-300 mV, larger by a factor of at least 4-5 than the widely known transmembrane potential (Wang, 2012). Because the electric field associated with the dipole potential is confined to the surface of the membrane, its strength is $10^{8}-10^{9} \mathrm{~V} / \mathrm{m}$, larger by $1-2$ orders of magnitude than the field associated with the transmembrane potential. Therefore, the dipole potential exerts significant effects on the conformation of transmembrane proteins (Clarke, 2015; Kovács et al., 2016; Zákány et al., 2019, 2020), on the binding of molecules to the membrane (Cladera \& O'Shea, 1998) and their transmembrane transport (Flewelling \& Hubbell, 1986). One of the most important factors determining the dipole potential is the sterol content of membranes. Cholesterol has been shown to increase the membrane dipole potential directly due to its intrinsic dipole moment and 
indirectly by increasing the order of lipids and interfacial water molecules and by changing the dielectric constant of the membrane (Sarkar et al., 2017; Starke-Peterkovic et al., 2006; Zákány et al., 2020). Due to this correlation, the dipole potential has been shown to be larger in raft-like membrane domains in cellular plasma membranes (Kovács et al., 2017). Statins, inhibitors of 3-hydroxy3-methyl-glutaryl-CoA (HMG-CoA) reductase, decrease the cholesterol content of cells in experimental and clinical settings and they were reported to decrease the dipole potential of the plasma membrane (Bjorkhem-Bergman et al., 2011; Sarkar et al., 2017). Statins are the most commonly used therapeutic agents to treat hypercholesterolaemia due to their beneficial effect on cardiovascular morbidity and mortality (Crismaru et al., 2020; Endo et al., 1977). Although adverse effects, for example myopathy, liver dysfunction and type 2 diabetes, have been associated with statins, they are usually well tolerated and successfully used even in combination (Schachter, 2005). Although the primary mechanism of action of all statins is identical, there are significant differences in their efficacy and bioavailability (Schachter, 2005). Atorvastatin is superior to other statins in requiring lower milligram equivalent doses to achieve the same effect on LDL-cholesterol levels (Jones et al., 1998). As opposed to simvastatin and lovastatin, which are pro-drugs of the active hydroxy-acid form, atorvastatin does not require enzymatic activation, a property not to be overlooked in in vitro applications (Corsin et al., 1995).

Corollary to the aforementioned principles, membrane potentials are expected to influence the uptake of penetratin due to the charged nature of the peptide. However, only a limited number of studies correlating electrostatic potentials and cell penetrating peptide uptake have been reported. Non-physiological abolishment of the transmembrane potential has been shown to inhibit the uptake of positively charged cell penetrating peptides (Rothbard et al., 2005). Although a negative dipole potential favours the incorporation of cell penetrating peptides into lipid monolayers in molecular dynamics simulations and in experiments (Via, Del Popolo, \& Wilke, 2018; Via, Klug, et al., 2018), such effects have not been described in lipid bilayers or living cells. Here, we not only show that the physiological, positive dipole potential of cellular membranes inhibits the uptake and endo-lysosomal escape of penetratin but also report that an artificial decrease of the dipole potentia and treatment with atorvastatin at concentrations corresponding to the clinical dose range stimulate entry of penetratin into the cytosol.

\section{2 | METHODS}

\section{1 | Synthesis and fluorescence labelling of penetratin}

Penetratin (RQIKIWFQNRRMKWKK-amide, MW $2245.75 \mathrm{~g} \cdot \mathrm{mol}^{-1}$ ) was synthesized manually on TentaGel R RAM (Rapp Polymere, Tübingen, Germany), a low crosslinked polystyrene PEG copolymer resin (the substitution was $0.2 \mathrm{mmol} \cdot \mathrm{g}^{-1}$ ) by the solid-phase method of Merrifield with standard Fmoc chemistry. All amino acid analogues were purchased from Iris Biotech (Marktredwitz, Germany). After coupling the last, $\mathrm{N}$-terminal amino acid, a 6-carbon linker (Fmoc-6-aminohexanoic acid, Iris Biotech) was added to the chain. This linker was used for reducing the potential effect of fluorescent dyes on penetratin. After removing the Fmoc protecting group, half of penetratin, still on the resin, was treated with 1.5 equivalents of AFDye532 N-hydroxysuccinimide ester (MW $723.77 \mathrm{~g} \cdot \mathrm{mol}^{-1}$, Fluoroprobes, Scottsdale, AZ, catalogue number: 1041) and 1.5 equivalents of diisopropylethylamine (DIPEA, Sigma-Aldrich, St. Louis, MO) in dimethylformamide (DMF, Sigma-Aldrich) overnight. The other half of penetratin was reacted with 1.5 equivalents of 5(6)-carboxynaphthofluorescein $\mathrm{N}$-succinimidyl ester (MW $573.51 \mathrm{~g} \cdot \mathrm{mol}^{-1}$, Setareh, Eugene, OR, catalogue number: 6964) in the presence of DIPEA in DMF. The labelled peptides were deprotected and released from the resin followed by filtration and precipitation. The crude products were purified by preparative reversed-phase HPLC (JASCO, Victoria, Canada) on a C18 column and lyophilized. The purity of the products (>95\%) was assessed by reversed-phase HPLC (JASCO) equipped with an analytical C18 column (Figure S1). The authenticity of each peptide was confirmed by Bruker electrospray ionization MS. The predicted and measured molecular mass of the $(\mathrm{M}+\mathrm{H})^{+}$form of AFDye532-penetratin was 2983.59 and 2983.548, respectively, whereas the predicted and measured molecular mass of the $(\mathrm{M}+\mathrm{H})^{+}$variant of NF-penetratin was 2833.33 and 2832.5, respectively. More detailed description of the synthesis and fluorescence labelling of penetratin is available in the supporting information section.

\section{$2.2 \quad$ Cell culture}

The SKBR-3 cell line (CLS Cat\# 300333/p3803_SK-BR-3,RRID: CVCL_0033) was obtained from the American Type Culture Collection (Manassas, VA), whereas MDA-MB-231 cells (NCI-DTP Cat\# MDA-MB-231, RRID:CVCL_0062) were a kind gift of Peter Bay (University of Debrecen). The cell lines were selected based on their different atorvastatin sensitivity (Göbel et al., 2019; Kimbung et al., 2016; Zhang et al., 2019). Both cell lines were propagated in DMEM containing 10\% FBS supplemented with antibiotics. Cells were harvested at a confluency of $80 \%-90 \%$ before measurements.

\section{3 | Treatment of cells with an inhibitor of endocytosis or lysosomal degradation}

In order to inhibit dynamin-dependent endocytic processes, cells were treated with $40 \mu \mathrm{M}$ of Dyngo4A (Abcam, Cambridge, UK; ab120689) for $30 \mathrm{~min}$ followed by the penetratin uptake experiment as without the inhibitor. For inhibiting lysosomal degradation, cells were treated with $30 \mu \mathrm{M}$ of the cysteine protease inhibitor, E-64d (aloxistatin, 
Sigma-Aldrich, E8640), for 30 min followed by incubating the cells in the presence of fluorescent penetratins.

\section{4 | Treatments modifying the dipole potential or inhibiting HMG-CoA reductase}

6-ketocholestanol (Sigma-Aldrich, K1250) and phloretin (SigmaAldrich, P7912) were used for measuring penetratin uptake at increased and decreased dipole potentials, respectively. Both compounds were applied at a concentration of $100 \mu \mathrm{M}$ at room temperature in the presence of $0.05 \%(\mathrm{v} / \mathrm{v})$ Pluronic F-127 (Sigma-Aldrich, P2443) for 10 min (Clarke \& Kane, 1997; Kovács et al., 2017). After the treatment, the dipole potential-modifying agents not incorporated into the membrane were removed by washing. In order to inhibit HMG-CoA reductase atorvastatin (Sigma-Aldrich, PZ0001) was applied at final concentrations of $1 \mathrm{nM}-10 \mu \mathrm{M}$ for $72 \mathrm{~h}$. The low nanomolar concentrations represent ranges found in human serum during atorvastatin treatment of patients with hypercholesterolaemia (Bjorkhem-Bergman et al., 2011).

\section{5 | Membrane dipole potential measurement with di-8-ANEPPS}

After treatment with atorvastatin, 6-ketocholestanol or phloretin cells were incubated with di-8-ANEPPS (Thermo Fisher, D3167) at a final concentration of $2 \mu \mathrm{M}$ on ice for 20 min (Clarke \& Kane, 1997; Gross et al., 1994; Kovács et al., 2017). Cells were kept on ice until measurement. Right before spectrofluorimetry, the sample was warmed to $37^{\circ} \mathrm{C}$ and spectra were acquired with a Fluorolog-3 spectrofluorimeter (Horiba Jobin Yvon, Edison, NJ). The temperature of the cuvette holder was adjusted to $37^{\circ} \mathrm{C}$ by a circulating water bath. Excitation spectra were registered between 410 and 520 nm, whereas the emission wavelength was set to $660 \mathrm{~nm}$ in order to minimize effects of membrane fluidity on the fluorescence of the indicator (Clarke \& Kane, 1997). The ratio of fluorescence intensities integrated between excitation wavelengths $410-416 \mathrm{~nm}$ and $503-510 \mathrm{~nm}$ correlates positively with the dipole potential (Clarke \& Kane, 1997; Kovács et al., 2017).

\section{6 | Measurement of membrane fluidity, hydration and cellular cholesterol content}

After treatment with atorvastatin, phloretin or 6-ketocholestanol, 150,000 cells were stained with $10 \mu \mathrm{M} 4^{\prime}$-(trimethylammonio)diphenylhexatriene (TMA-DPH) or $2 \mu \mathrm{M}$ Laurdan (6-dodecanoyl- $N, N$ dimethyl-2-naphthylamine; both obtained from Sigma-Aldrich) for $30 \mathrm{~min}$ at room temperature. Anisotropy of TMA-DPH was measured with a Fluorolog-3 spectrofluorimeter with the temperature of the cuvette holder adjusted to $37^{\circ} \mathrm{C}$. TMA-DPH was excited at $352 \mathrm{~nm}$ and its emission was measured at $430 \mathrm{~nm}$. Fluorescence anisotropy $(r)$ was measured in the L-format according to the following formula (Kuhry et al., 1983):

$$
r=\frac{I_{\mathrm{vv}}-G I_{\mathrm{vh}}}{I_{\mathrm{vv}}+2 G I_{\mathrm{vh}}}
$$

where $I_{\mathrm{vv}}$ and $I_{\mathrm{vh}}$ are the vertical and horizontal components, respectively, of the fluorescence excited by vertically polarized light and $G$ is an instrument-specific correction factor characterizing the different sensitivity of the detection system for vertically and horizontally polarized light. Laurdan was excited at $350 \mathrm{~nm}$ and its emission was detected in the blue range of its emission spectrum between 430-440 nm (I $\left.I_{\text {blue }}\right)$ and at the red edge between 495-505 nm (I $\left.I_{\text {red }}\right)$ to measure generalized polarization (GP) according to the following formula (Parasassi et al., 1991):

$$
G P=\frac{I_{\text {blue }}-I_{\text {red }}}{I_{\text {blue }}+I_{\text {red }}}
$$

Total cholesterol content of cellular samples was determined using Cholesterol Quantification Kit (MAK043, Sigma-Aldrich).

\subsection{Flow cytometric measurement of penetratin uptake and endo-lysosomal release}

Cells were incubated in the continuous presence of $5 \mu \mathrm{M}$ AFDye532-penetratin, $5 \mu \mathrm{M}$ NF-penetratin and $0.25 \mu \mathrm{g} \cdot \mathrm{ml}^{-1}$ DAPI (Sigma-Aldrich, D9564) at $37^{\circ} \mathrm{C}$ in the thermostated sample holder of a Becton Dickinson FACSAria III flow cytometer (Becton Dickinson, Mountain View, CA). DAPI was excited at $405 \mathrm{~nm}$ and its emission was measured between 430 and $470 \mathrm{~nm}$. The fluorescence of AFDye532 was excited at $488 \mathrm{~nm}$ and detected between 515 and $545 \mathrm{~nm}$. The excitation wavelength and detection wavelength ranges for NF were $561 \mathrm{~nm}$ and 663-677 nm, respectively. Apart from background measurements, every experiment was carried out for 20 min and the measurement started right after adding the DAPI/penetratin mixture, preheated to $37^{\circ} \mathrm{C}$, to the cells. After spectral compensation and gating out dead cells based on DAPI positivity, the time-correlated fluorescence intensities were exported using FCS Express (De Novo Software, Pasadena, CA). A custom-written Matlab (RRID: SCR_001622) program was used for calculating a moving average with a window size of $20 \mathrm{~s}$. The $\mathrm{pH}$-insensitive fluorescence of AFDye532 is proportional to the total cellular uptake of penetratin. Due to quenching of $\mathrm{NF}$ at acidic $\mathrm{pH}$, the ratio of NF-penetratin to AFDye532-penetratin fluorescence intensities reports the endo-lysosomal escape of the cell penetrating peptide (Qian et al., 2015). The fluorescence intensities of both indicators were normalized to the mean intensity measured in the first-time window. This normalization was required to reduce within-group variability due to differences between initial fluorescence intensities, which would have obscured between-group differences. Normalized mean intensities were divided by each other to obtain the NF-penetratin/AFDye532-penetratin ratio. The SEM of the ratio parameter was calculated using error propagation analysis assuming independence of the two fluorescence intensities: 


$$
S E M_{\text {ratio }}=\sqrt{\frac{\overline{N F}^{2}}{A F D \bar{y} e 532^{4}} S E M_{A F D y e 532}{ }^{2}+\frac{1}{A F D \overline{y e} 532^{2}} S E M_{N F}^{2}}
$$

$\overline{N F}$ and $A F D \bar{y}$ 533 designate the mean intensity of the respective parameter.

\section{8 | Investigation of penetratin uptake by confocal microscopy}

Penetratin uptake and endo-lysosomal release was visualized using a Zeiss LSM880 confocal microscope (Carl Zeiss, Oberkochen, Germany). Fluorescently labelled penetratins were used at the same concentration as in flow cytometry. The temperature of the microscope stage was adjusted to $37^{\circ} \mathrm{C}$. AFDye 532 and NF were excited at 514 and $633 \mathrm{~nm}$, respectively. Detection of AFDye 532 and NF fluorescence was carried out between 525-629 nm and 637-759 nm, respectively.

\section{9 | Data handling and statistical analysis}

Experiments were performed in an unblinded way because the experimenter must know about the experimental conditions and to avoid excessive complexity in labelling of reagents. However, primary analysis of experimental data was blinded since analyses and the experiments were performed by different persons and the analyser was unaware of the experimental conditions. Randomization is irrelevant in the case of this study because all compared samples originated from the same culture flask. However, treatments were carried out in different order to eliminate differences due to timing of experiments. Multiple comparisons were performed with ANOVA. If the between-group differences were found to be significant $(p<.05)$ without significant variance inhomogeneity, post hoc comparisons were carried out with Tukey's HSD test. Statistical calculations were carried out with customwritten routines in Excel (RRID:SCR_016137) and Matlab (RRID: SCR_001622). Group sizes always refer to biological replicates. If results are presented as bar charts, the distribution of individual experimental results did not reveal unusual aspects of the data not obvious from the bar chart. The data and statistical analysis comply with the recommendations of the British Journal of Pharmacology on experimental design and analysis in pharmacology (Curtis et al., 2018).

\subsection{Nomenclature of targets and ligands}

Key protein targets and ligands in this article are hyperlinked to corresponding entries in the IUPHAR/BPS Guide to PHARMACOLOGY http://www.guidetopharmacology.org and are permanently archived in the Concise Guide to PHARMACOLOGY 2019/20 (Alexander et al., 2019).

\section{3 | RESULTS}

\subsection{Fluorescence labelling reveals cellular uptake and delayed endo-lysosomal escape of penetratin}

Penetratin is one of the most widely used cell penetrating peptides (Xie et al., 2020). The fact that it outperforms other cell penetrating

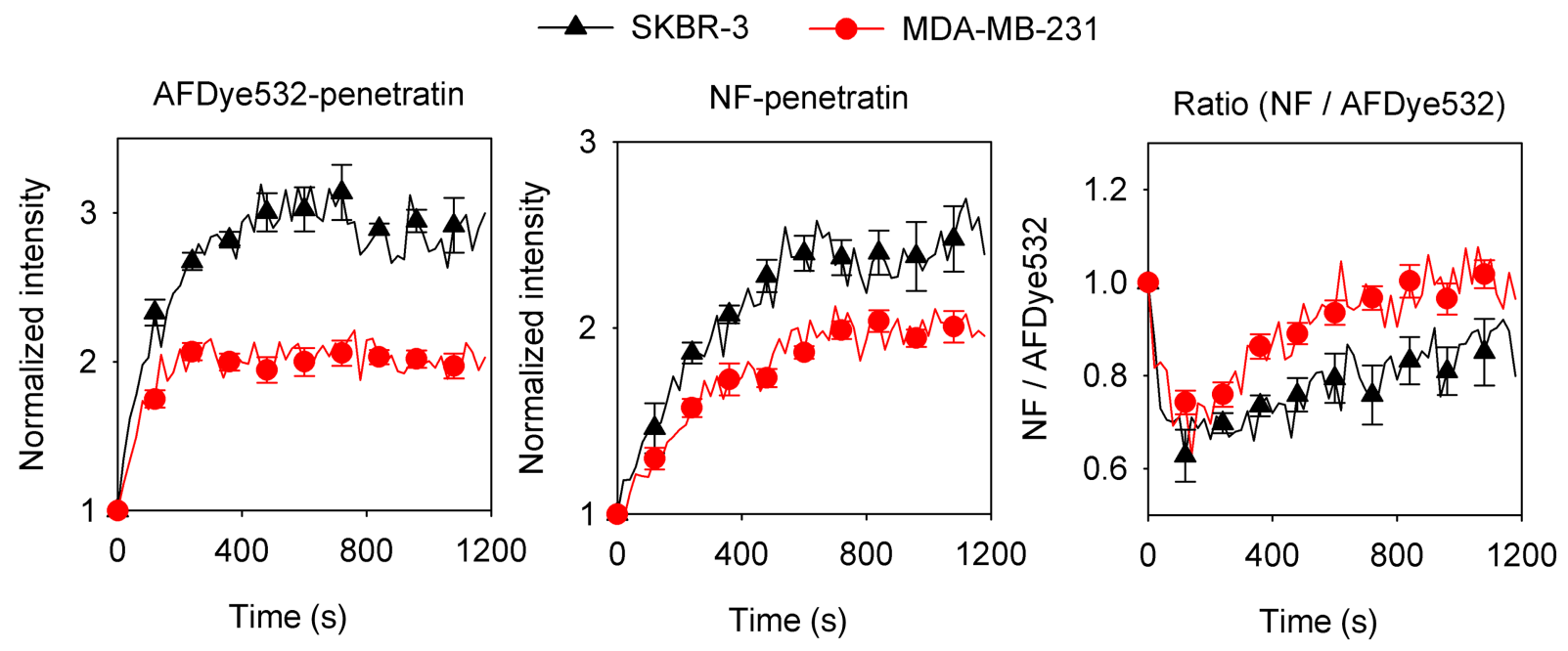

FIGURE 1 Cellular uptake and endo-lysosomal escape of penetratin. Two different cell types were incubated at $37^{\circ} \mathrm{C}$ in the continuous presence of fluorescently labelled penetratins (5 $\mathrm{M}$ AFDye 532-penetratin and $5 \mu \mathrm{M}$ NF-penetratin). Time-correlated flow cytometric recording of the fluorescence intensity of cells was performed. The average intensity of AFDye532-penetratin, characterizing total cellular uptake, and the average intensity of NF-penetratin, characteristic of the amount of penetratin present in pH-neutral compartments, were calculated as a function of time after gating out debris and non-viable cells. The ratio of the two fluorescence intensities is plotted in the panel on the right. The continuous lines show the average fluorescence intensity calculated for 20-s time periods normalized to the intensity in the first-time window. The symbols with error bars, representing the SEM calculated from 13-15 samples from five biological replicates, are only shown for every sixth data point for clarity 
peptides in certain respects prompted us to use it in our model system (Liu et al., 2014). Penetratin was labelled with two different fluorescent dyes in order to study the mechanism and kinetics of its cellular uptake. The fluorescence of naphthofluorescein (NF) is quenched at acidic $\mathrm{pH}$ enabling studying the release of penetratin from the acidic endo-lysosomal compartment (Qian et al., 2015). In order to complement the information on endo-lysosomal escape, we chose labelling with AFDye532, a dye exhibiting $\mathrm{pH}$-independent fluorescence to report on the total cellular content of penetratin. The cellular fluorescence of AFDye532-penetratin is proportional to the total cellular uptake of penetratin. The intensity of NF-penetratin characterizes its concentration in non-acidic compartments (mainly the cytosol), whereas the ratio of NF-labelled and AFDye532-labelled penetratin intensities reveals the fractional escape of the cell penetrating peptide from acidic compartments. The $\mathrm{MW}$ and purity of labelled penetratin were checked by MS and high-performance LC (Figure S1). We developed an approach based on flow cytometric measurement of cell-associated fluorescence signals to separately measure the kinetics of cellular uptake and endo-lysosomal release of these fluorescently labelled penetratins. Cells were incubated in the continuous presence of an equimolar mixture of NF-penetratin and AFDye532-penetratin ( $5 \mu \mathrm{M}$ of each) at $37^{\circ} \mathrm{C}$, and the fluorescence intensity of cells was measured in a time-correlated manner. After removing cells with compromised membrane permeability based on DAPI staining from the dataset and compensating for spectral crosstalk, the time-dependent change of fluorescence intensity was plotted using moving average smoothing. The signal of AFDye532-penetratin, corresponding to the total cellular content of penetratin, reached saturation at $200-400 \mathrm{~s}$ in two different cell lines, whereas a significantly delayed saturation of penetratin concentration in the neutral, cytoplasmic compartment at $800-1000 \mathrm{~s}$ was observed based on the $\mathrm{pH}$-sensitive fluorescence of NF-penetratin (Figure 1). Two aspects of the fluorescence traces imply that endocytosis plays a fundamental role in penetratin uptake:- (i) the ratio of NF to AFDye532 intensities, characterizing the fraction of penetratin in neutral compartments, initially declined, corresponding to a preferential presence of penetratin in acidic endosomes and (ii) both NF intensity and the NF-penetratin/ AFDye532-penetratin ratio reach saturation long after the total cellular content of penetratin, reported by AFDye532-penetratin intensity, is saturated (Figure 1). This latter feature is the consequence of penetratin leaving acidic compartments leading to unquenching of NF fluorescence.

In order to reveal the contribution of endocytosis to penetratin uptake, experiments were also performed in the presence of Dyngo4a, an inhibitor of dynamin (McCluskey et al., 2013). Comparison to untreated cells showed that the inhibitor brought about a statistically significant, $50 \%-80 \%$ decrease in the total cellular content and cytoplasmic concentration of the fluorescent penetratins (Figure S2). Although inhibition of dynamin may not block all kinds of endocytic processes, these experiments argue for a dominant role of endocytosis in penetratin uptake.

Endocytic uptake and the presence of penetratin in the endo-lysosomal compartment run the risk of degradation (Fischer et al., 2010), even though penetratin escapes efficiently from this compartment. We feared that what appears to be cytosolic accumulation of penetratin based on the increase in naphthofluorescein fluorescence may actually be due to increased concentration of free dye or penetratin fragments in the cytoplasm. Although free AFDye532 does not penetrate across the plasma membrane, the permeability of the plasma membrane to both the anionic and neutral isomers of free naphthofluorescein is relatively high (Figure S3). These experiments show that free naphthofluorescein could indeed escape from the endo-lysosomal compartment if generated in substantial quantities, a circumstance that would complicate the interpretation of results. In order to establish the contribution of lysosomal degradation to the increase in naphthofluorescein fluorescence, we carried out the penetratin uptake experiments in the presence of aloxistan (E-64d), an inhibitor of lysosomal cysteine proteases. The experiments showed
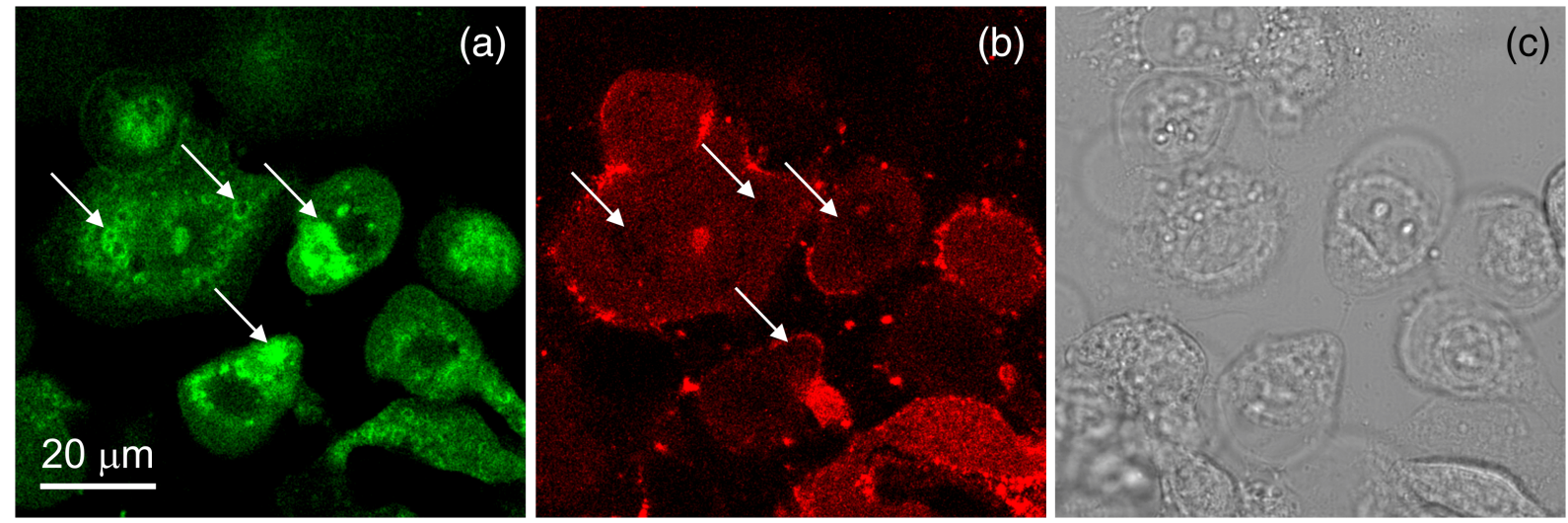

FIGURE 2 Confocal microscopic images of live cells dual-labelled with AFDye532- and NF-penetratin. Cells were incubated with AFDye 532-penetratin (a) and NF-penetratin (b) at $37^{\circ} \mathrm{C}$ for $20 \mathrm{~min}$ and images were captured by confocal microscopy. Panel (c) displays a differential interference contrast image of the cells. The arrows in the fluorescence images point at areas showing the anti-correlation between the $\mathrm{pH}$-insensitive fluorescence of AFDye532 and the fluorescence of $\mathrm{NF}$ quenched at acidic $\mathrm{pH}$ 
that degradation of penetratin does not play a significant role in the fluorescence intensity changes observed within the 20-min time window of our experiments (Figure S4). We believe that longer incubation times applied in other studies (e.g. $2 \mathrm{~h}$ in Fischer et al., 2010) might be required for substantial lysosomal degradation of cell penetrating peptides to occur.

The elimination of cells with compromised membrane permeability turned out to be crucial. Whereas the instantaneous binding of penetratin to cells with increased membrane permeability was much higher than in intact cells, the fold-increase in the time-dependent penetratin uptake of these cells was smaller than in intact ones (Figure S5). This finding reveals the advantage of fluorescence-based analysis for the reliable measurement of penetratin uptake of viable cells. On the other hand, it must also be pointed out that the fluorescence label itself may also influence the binding properties of penetratin (Christensen et al., 2019). However, flow cytometric or other fluorescence techniques surpass label-free approaches, such as NMR or MS (Burlina et al., 2005; Christensen et al., 2019), in their flexibility to be combined with the measurement of other cellular parameters (e.g. membrane permeability). Results obtained using fluorescence techniques in this manuscript must be interpreted with the pros and cons of fluorescence techniques in mind.

Because flow cytometry lacks subcellular resolution, we correlated the fluorescence intensities of the two reporters with their cellular location. Confocal microscopic investigation of the distribution of the fluorescent penetratin derivatives revealed that AFDye532-penetratin exhibits bright fluorescence even in endosomes where the fluorescence of NF is quenched and that the cellassociated fluorescence of NF originates from outside the endolysosomal compartment (Figure 2). This observation confirms that interpretation of the fluorescence intensity of AFDye532-penetratin and NF-penetratin, measured by flow cytometry, as total cellular penetratin uptake and the amount outside endosomes, respectively, is indeed correct. Because AFDye532 and NF form a Förster resonance energy transfer pair, anticorrelation between their fluorescence intensities could also have been caused by energy transfer, that is, a high local concentration of the acceptor (NF) quenching the fluorescence of the donor (AFDye532). In order to exclude this possibility, the equimolar mixture of fluorescent penetratins was supplemented with $10 \mu \mathrm{M}$ unlabelled penetratin. If energy transfer is to blame for the anti-correlation between the fluorescence intensities of the two dyes, dilution of their local concentration with unlabelled penetratin is expected to eliminate or reduce this anticorrelation. However, the time dependence of the fluorescence intensities and their ratio were not changed significantly by the presence of unlabelled penetratin allowing us to conclude that the fluorescence intensities of AFDye532 and NF correctly report total cellular uptake of penetratin and its concentration in neutral compartments, respectively (Figure S6). In summary, we established a flow cytometric approach revealing the different kinetics of cellular uptake and endo-lysosomal release of penetratin setting the stage for further analysis of the effect of dipole potential-modifying agents on penetratin uptake.
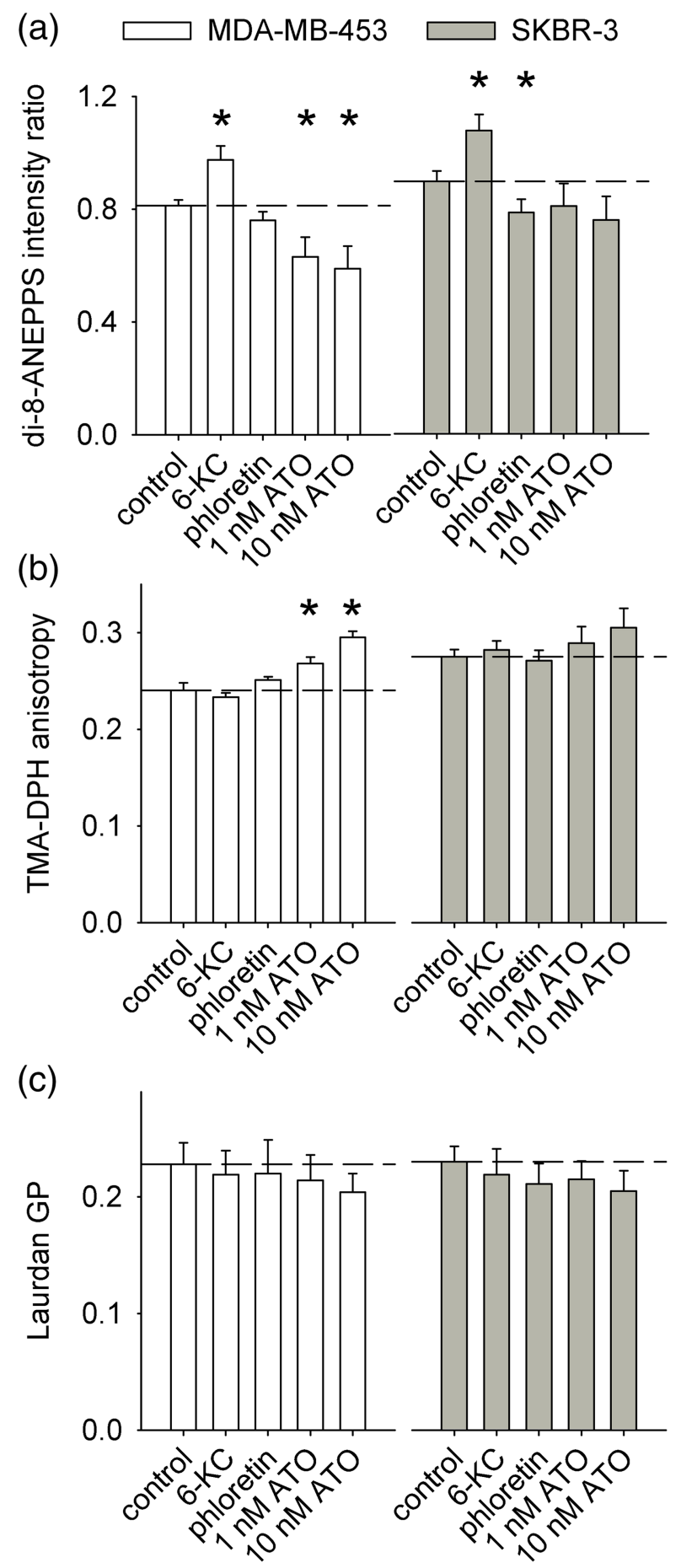

FIGURE 3 Characterization of the effects of phloretin, 6-ketocholestanol and atorvastatin on the biophysical properties of the membrane. Cells were pretreated with phloretin, 6-ketocholestanol (6-KC) or two different concentrations of atorvastatin (ATO) as described in materials and methods. The dipole potential was measured with di-8-ANEPPS, whose excitation intensity ratio correlates positively with the dipole potential (a). The fluorescence anisotropy of TMA-DPH is inversely related to membrane fluidity (b), whereas the generalized polarization (GP) of Laurdan is proportional to the compactness of the membrane (c). The error bars display the SEM of five biological replicates. Asterisks indicate statistical significance $(P<.05)$ reported by ANOVA followed by Tukey's HSD test 


\section{2 | Reduction of the membrane dipole potential enhances uptake and endo-lysosomal release of penetratin}

Because the strong intramembrane dipole potential is expected to affect the uptake and/or endo-lysosomal release of positively charged penetratin, we treated cells with 6-ketocholestanol and phloretin, agents known to increase and decrease, respectively, the positive, intramembrane dipole potential (Gross et al., 1994; Kovács et al., 2016). Spectrofluorometric analysis of the fluorescence intensity ratio of the dipole potential sensitive dye, di-8-ANEPPS, confirmed that 6-ketocholestanol significantly increased the dipole potential. On the other hand, phloretin decreased the dipole potential although its effect did not reach statistical significance in one of the cell lines (Figure 3a). The effect of phloretin is always on the verge of statistical significance (in the current case $p=.07$ ). Power analysis predicted that a sample size of $\geq 10$ would be required for firm statistical significance using the usual effect size of phloretin and type I and type II error probabilities of .05 and .2, respectively. Given the large number of different treatment conditions, such large samples were not possible. However, the significance of the effect of the dipole potential-modifying treatments on the dipole potential in ANOVA and the correlation between the dipole potential and penetratin uptake (to be discussed later) argue for the existence of an effect of the treatments on the dipole potential. Because none of the agents compromised cell viability (Figure S7), these observations confirm our previous results regarding the effect of phloretin and 6-kethocholestanol on the dipole potential (Kovács et al., 2016).

Having established that the treatments modify the dipole potential according to expectations, we set out to characterize the effect of an altered dipole potential on the uptake of penetratin. A diminished dipole potential significantly enhanced both the total cellular uptake of penetratin and its concentration in non-acidic compartments in both SKBR-3 and MDA-MB-231 cells (Figure 4). Because both of these processes were enhanced at the lower dipole potential achieved by phloretin treatment, the ratio of the NF and AFDye532 intensities, characterizing the fractional release from acidic compartments, remained unchanged (correlations between penetratin uptake and the dipole potential will be further analysed in a subsequent section and in Figure S9). As far as the most significant measure of penetratin

- Control $-\mathrm{O}$ - Phloretin (decreased dipole pot.) 6-ketocholestanol (increased dipole pot.)
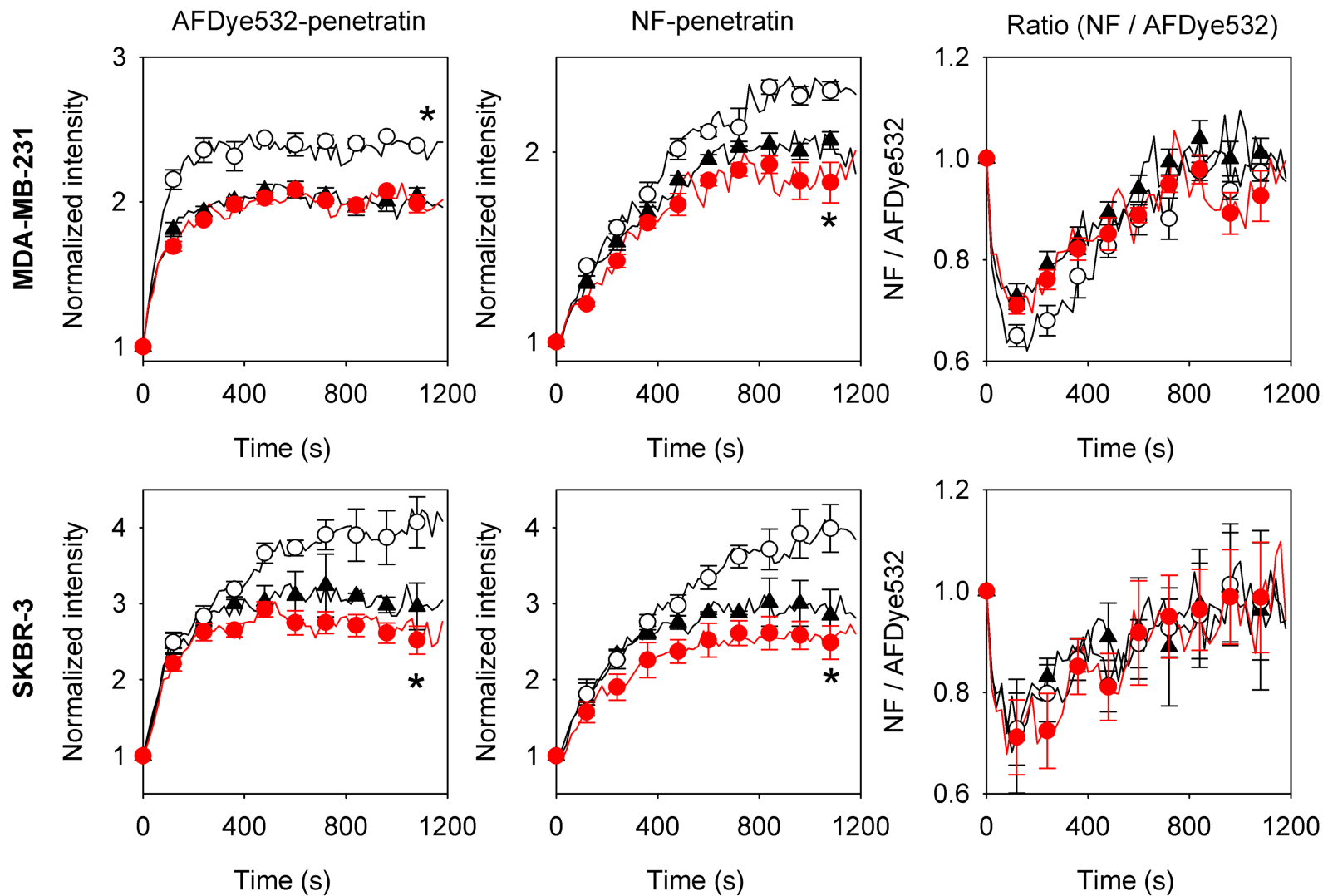

FIG URE 4 Decreased dipole potential enhances cellular uptake and endo-lysosomal escape of penetratin. Cells (SKBR-3, MDA-MB-231, displayed on the left) were treated with a mixture of AFDye532-penetratin and NF-penetratin at $37^{\circ} \mathrm{C}$, and their fluorescence intensity was measured by flow cytometry. The membrane dipole potential of cells was decreased and increased by pretreatment with phloretin and 6-ketocholestanol, respectively. Time-dependent intensities and their ratio were determined after gating out debris and dead cells. The error bars represent the SEM calculated from 12-14 samples from five biological replicates. Asterisks indicate significant difference of the phloretin-treated sample compared with the control at $20 \mathrm{~min}(P<.05)$ 
uptake, its concentration in the cytosol (reported by NF-penetratin intensity) is concerned, phloretin achieved a 50\% increase in this parameter. On the other hand, 6-ketocholestanol, which significantly increased the dipole potential (Figure 3a), had a statistically nonsignificant effect on both the total cellular uptake and endo-lysosomal release of penetratin (Figure 4). The presence of an initial decline in the NF-penetratin/AFDye532-penetratin intensity ratio in control samples and in samples with an altered dipole potential and the protracted saturation of NF-penetratin fluorescence in all samples suggest that the uptake mechanism of penetratin, to which endocytosis contributes significantly, was not altered by any of the treatments. We also established that the contribution of lysosomal degradation of penetratin to its accumulation in the cytosol is negligible under the condition of altered dipole potential as well (Figure S4). In conclusion, we have shown that the physiological level of the intramembrane, positive dipole potential significantly inhibits the uptake of penetratin.

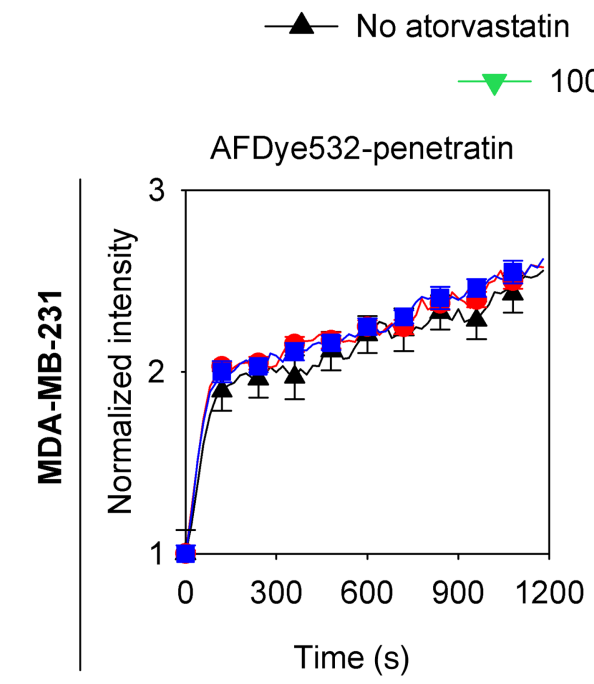

$-1 \mathrm{nM}$ atorvastatin

$-10 \mathrm{nM}$ atorvastatin $00 \mathrm{nM}$ atorvastatin

$\multimap 10 \mu \mathrm{M}$ atorvastatin
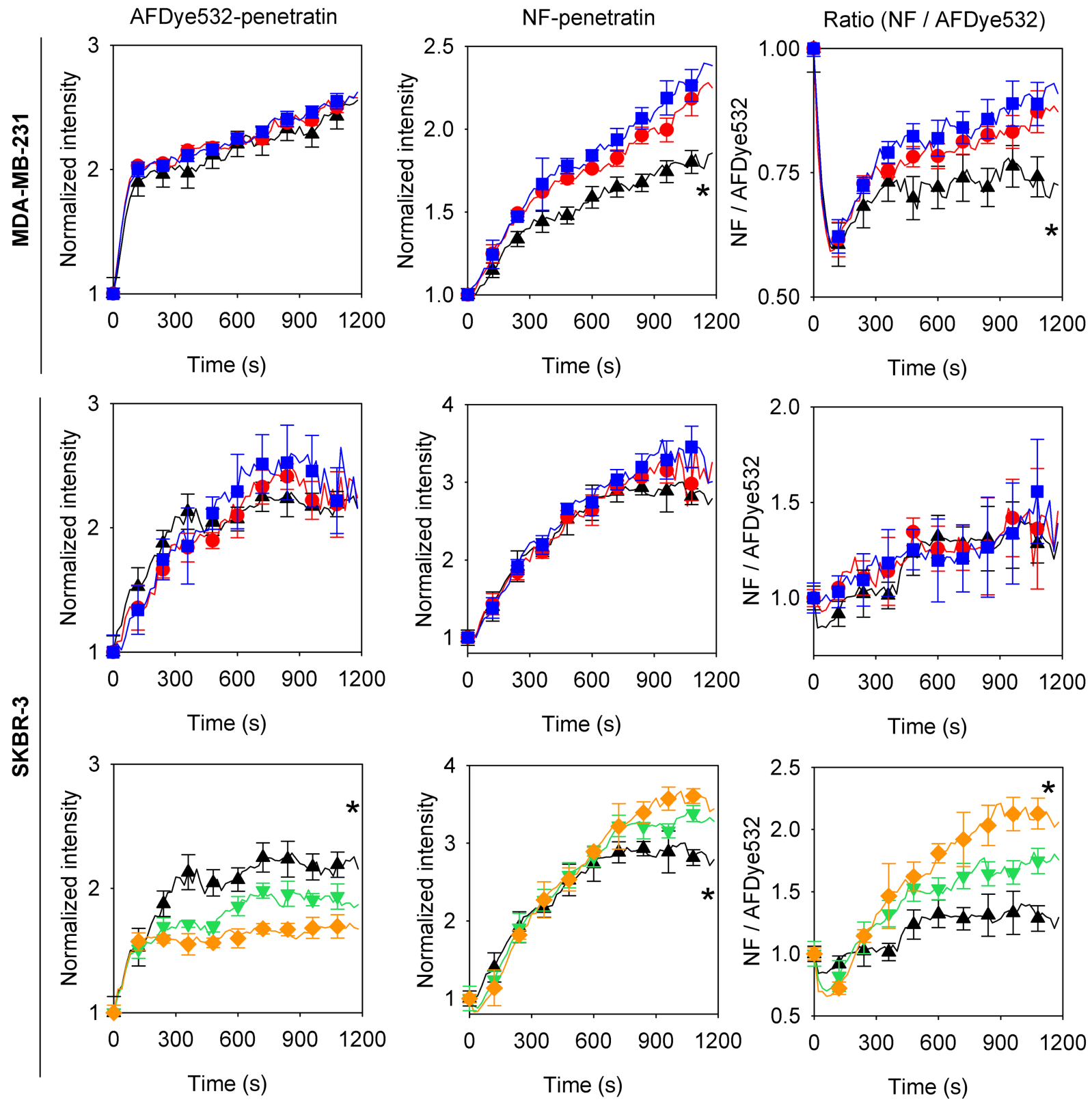

FIGURE 5 Atorvastatin enhances endo-lysosomal escape of penetratin. Two different cell types were pretreated with the indicated concentrations of atorvastatin for $72 \mathrm{~h}$ and the uptake of AFDye-532-penetratin and NF-penetratin was followed by flow cytometry. Timedependent intensities and their ratio were determined after gating out debris and dead cells. The error bars represent the SEM calculated from 13-15 samples from five biological replicates. Asterisks indicate significant difference of the sample treated with the highest concentration of atorvastatin in the figure compared with the control at $20 \mathrm{~min}(P<.05)$ 


\section{3 | Statins increase the endo-lysosomal release of penetratin due to decreased dipole potential}

Increasing the uptake of cell penetrating peptides has great potential medical benefit. Because the treatment used for decreasing the dipole potential in the previous section cannot be applied in humans, we sought an alternative approach to enhance the uptake of penetratin by modulating the dipole potential. The dipole potential correlates with membrane cholesterol content (Kovács et al., 2017; Sarkar et al., 2017) and statins are known to decrease the dipole potential (Sarkar et al., 2017). We opted for atorvastatin because it is one of the most effective statins and it is the active substance not requiring enzymatic activation (Corsini et al., 1995; Jones et al., 1998; Schaefer et al., 2004). Atorvastatin, used at a concentration of 1-10 $\mathrm{nM}$ corresponding to the serum concentration in human patients (Bjorkhem-Bergman et al., 2011), significantly decreased the total cholesterol content of MDA-MB-231 cells by $40 \%-50 \%$. Albeit to a lesser extent, atorvastatin also decreased the cholesterol content of SKBR-3 cells (Figure S8). In perfect agreement with these results, atorvastatin decreased the dipole potential in both cell lines, but SKBR-3 displayed lower sensitivity (Figure 3a). Treatment of MDA-MB-231 cells with the same concentration range of atorvastatin significantly enhanced the endo-lysosomal release of penetratin with only a marginal effect on total cellular uptake (Figure 5). At the same time, the effect of atorvastatin on SKBR-3 cells was smaller and it did not reach statistical significance. Because the extent of decrease in the cholesterol content of this cell line turned out to be smaller compared with MDA-MB-231, we also tested the effect of higher atorvastatin concentrations; $100 \mathrm{nM}$ and $10 \mu \mathrm{M}$ of atorvastatin decreased the total cellular uptake of penetratin in SKBR-3 cells. We attribute this finding to either endocytosis inhibited by statin treatment (Sidaway et al., 2004; Yilmaz et al., 2006) or to compromised cell viability even though cells with increased membrane permeability (DAPIpositive cells) were discarded from the analysis. However, the amount of penetratin leaving the endo-lysosomal compartment was significantly higher in cells treated with these high atorvastatin concentrations even though the total cellular uptake was lower (Figure 5). This finding is evidenced by the almost two times higher NF/AFDye532 intensity ratio characterizing the fraction of penetratin escaping from endosomes. The per cent increase in the cytosolic concentration of penetratin achieved by statin treatment $(10 \mathrm{nM}$ and $10 \mu \mathrm{M}$ in the case of MDA-MB-231 and SKBR-3 cells, respectively) was approximately $50 \%$ and $30 \%$ for the MDA-MB-231 and SKBR-3 cell line, respectively. None of the atorvastatin concentrations changed the fact that the fluorescence intensity of AFDye532-penetratin saturated much sooner than that of NF-penetratin, implying that endocytosis remained a significant contributor to penetratin uptake. The contribution of lysosomal degradation of penetratin to the buildup of its cytosolic concentration was negligible after atorvastatin treatment as well (Figure S4). Because cholesterol depletion might also affect cell viability and membrane permeability, it was essential to show that none of the treatments had any significant effect on cell viability (Figure S7). In conclusion, we have convincingly shown that release of penetratin from the endo-lysosomal compartment is the step that is the most significantly increased by atorvastatin treatment.

\subsection{Characterization of the effects of atorvastatin and the dipole potential modifying compounds on membrane biophysical properties}

The previous experiments revealed that treatments reducing the membrane dipole potential enhance the total cellular uptake or the release of penetratin from endosomes, while increasing the dipole potential results in an opposite, weak effect. The validity of this conclusion was checked by calculating the rank-correlation between the dipole potential and penetratin uptake (Figure S9). A significant, negative correlation was revealed between the dipole potential and the total uptake (AFDye532 fluorescence), the concentration of penetratin in non-acidic compartments (NF fluorescence) and the fractional release of penetratin from the endo-lysosomal compartment (ratio of NF and AFDye532 intensities). Because the experimental data corresponding to the effect of phloretin on total cellular uptake of penetratin seemed to deviate from the trend established by the other conditions, the correlation was also tested by removing these outlier data points. The significant and biologically relevant negative correlation between the endo-lysosomal release of penetratin and the dipole potential was retained. However, removing the data points corresponding to phloretin treatment eliminated most of the dipole potential-dependent changes in total cellular uptake (Figure S9). This observation indicates that although both atorvastatin and phloretin decrease the dipole potential, their cellular effects and their influence on penetratin uptake are different. Because our measurement approach based on the $\mathrm{pH}$-dependent fluorescence of NF assumes that the $\mathrm{pH}$ of the endo-lysosomal compartment is equally acidic in all compared samples, a possible explanation for the different results obtained with atorvastatin and phloretin is that they alter the $\mathrm{pH}$ of lysosomes. Therefore, we performed fluorescence ratiometric measurements of lysosomal $\mathrm{pH}$, which revealed no significant effect of any of the treatments on lysosomal $\mathrm{pH}$ (Figure S10). These results imply that the ratio of the fluorescence of AFDye532-penetratin and NF-penetratin correctly reports the release of penetratin from acidic compartments. The issue of the different effect of phloretin and atorvastatin on penetratin release will be further considered in Section 4.

Because treatments altering the dipole potential could potentially also modify other biophysical properties of the membrane, the correlations between penetratin uptake and two other fluorescent indicators were also tested. Membrane fluidity, inversely proportional to microviscosity and the fluorescence anisotropy of TMA-DPH (Kuhry et al., 1983), was not changed by any of the treatments in the SKBR-3 cell line and phloretin and 6-ketocholestanol were also without effect in MDA-MB-231 cells (Figure 3b). In contrast, an unexpected decrease in the membrane fluidity of this cell line was induced by atorvastatin, an effect most likely attributable to the compensatory increase in the synthesis of certain lipid species in order to 
compensate for inhibited cholesterol synthesis (Dawaliby et al., 2016). Membrane fluidity and total cellular uptake of penetratin did not correlate strongly with each other, but there was a strong correlation between membrane fluidity and the fractional escape of penetratin from acidic compartments (Figure S9). The positive correlation coefficient implies that release of penetratin from the endo-lysosomal compartment is more efficient across less fluid membranes. However, the power of membrane fluidity to predict endo-lysosomal escape of penetratin is limited by the fact that none of the treatments induced any significant change in the membrane fluidity of SKBR-3 cells, whereas phloretin increased endosomal release of penetratin in this cell line.

The effect of the treatments on the generalized polarization of Laurdan, known to be proportional to membrane compactness and inversely related to membrane hydration (Parasassi et al., 1991), was also tested. None of the conditions led to any significant change in membrane compactness in the two cell lines (Figure 3c). Generalized polarization of Laurdan was weakly correlated with total penetratin uptake or its concentration in non-acidic compartments (with $p$ values close to the limit of statistical significance), but the biological relevance of this correlation is undermined by the very small changes in generalized polarization (Figure S9). Although membrane fluidity was strongly correlated with the fractional release of penetratin from the endo-lysosomal compartment, the dipole potential correlated with all three measures of penetratin uptake. Given the higher number of significant correlations between penetratin uptake and the dipole potential, and the inability of membrane fluidity to predict the phloretin-induced enhancement of penetratin uptake in SKBR-3 cells, we can conclude that the dipole potential has the largest power for predicting penetratin uptake and its escape from endosomes.

\section{DISCUSSION}

In this study, correlative measurement of the biophysical properties of the plasma membrane and the uptake of penetratin allowed us to reach the following major conclusions:- (i) the physiological, positive membrane dipole potential inhibits the total cellular uptake of penetratin and its release from acidic endo-lysosomes. This conclusion is based on temporal measurement of the cellular intensity of AFDye532-labelled and NF-labelled penetratin, respectively. The effect of the dipole potential on escape of penetratin from the endolysosomal compartment is most likely mediated by an alteration in the membrane insertion of the cell penetrating peptide, because incorporation and penetration of peptides and small hydrophobic molecules into the membrane are known to depend on the dipole potential (Cladera \& O'Shea, 1998). Although endocytosis itself is less likely to be affected significantly by the dipole potential, the amount of penetratin taken up by endocytosis is still most likely modified by the dipole potential due to the altered binding of the peptide to the plasma membrane from which endocytic vesicles are formed. (ii) Treatment with phloretin and atorvastatin reduced the membrane dipole potential and led to a significantly enhanced cytoplasmic concentration of penetratin. Because the applied, nanomolar concentration of atorvastatin is identical to that used in the clinical setting, the finding of statin-boosted uptake of penetratin is of potential medical significance. (iii) By analysing the correlation between several membrane biophysical properties and penetratin uptake the dipole potential turned out to be the only characteristic of sufficient value for predicting penetratin uptake. The fact that penetratin is a positively charged peptide not only provides an obvious explanation for why the positive, intramembrane dipole potential presents a barrier for the membrane crossing of this peptide, but it also suggests that these principles may also apply to other cationic cell penetrating peptides.

Although both phloretin and atorvastatin decreased the dipole potential and increased penetratin uptake, their differential effects raise a question about which step in the uptake process is the most sensitive to the dipole potential. In particular, although atorvastatin decreased the dipole potential more substantially than phloretin, only the latter induced an increase in the total cellular uptake of penetratin, whereas atorvastatin only increased the escape from acidic endo-lysosomes. These apparent contradictions can be resolved by the following three points. (i) The fact that phloretin does not increase the fraction of penetratin released from the endo-lysosomal compartment is explained by the fact that this compound is unlikely to reach sufficient concentrations in intracellular membranes during the brief, 10-min incubation and therefore the dipole potential of these compartments remains largely unaffected. On the other hand, the 3-day treatment with atorvastatin is sufficiently long so that substantial decrease in the cholesterol content and consequent reduction in the dipole potential of endo-lysosomal membranes can take place. (ii) The minuscule, phloretin-induced decrease of the dipole potential and the significantly elevated uptake of penetratin after phloretin treatment also require clarification. We assume that the physiological level of the dipole potential already limits penetratin uptake as much as potentially achievable by the dipole potential. This conclusion is supported by the fact that the significantly enhanced dipole potential after 6-ketocholestanol treatment had hardly any effect on the characteristics of penetratin uptake. On the other hand, even the relatively minor decrease in the dipole potential, achieved by phloretin, may be sufficient to facilitate penetratin uptake. (iii) Although the reduction in the dipole potential achieved by atorvastatin is significantly larger than the phloretin-induced change, atorvastatin had no effect on the total cellular concentration of penetratin. The sudden rise in AFDye532-penetratin intensity and the protracted increase in the intensity of NF-penetratin indicate that initial uptake is mediated by endocytosis, a characteristic left unaltered by any of the treatments. Therefore, the lack of statin-induced increase in the total uptake of penetratin implies that endocytosis must be hindered in atorvastatin-treated samples. Indeed, it has been shown that statins inhibit endocytosis by interfering with the prenylation-dependent function of certain G proteins (Sidaway et al., 2004; Yilmaz et al., 2006). Alternatively, the compensatory increase in membrane viscosity after atorvastatin treatment may also impede endocytosis.

Despite the aforementioned uncertainties, two of the treatments, phloretin and atorvastatin, significantly enhanced the concentration of penetratin in the cytosol, the compartment most relevant from a 
therapeutical point of view. Analysis of the correlation between penetratin uptake and different biophysical properties of the membrane revealed that the dipole potential is the strongest predictor of penetratin uptake. Although the dipole potential, membrane compactness and viscosity characterize different membrane properties, all of them are related to membrane order. Therefore, it is not surprising that besides the dipole potential, other membrane characteristics also correlate with penetratin uptake. Membrane viscosity is correlated with endo-lysosomal release of penetratin. However, the predictive value of this correlation is undermined by the following two points:(i) although phloretin exerts no effect on membrane fluidity, it significantly enhances penetratin uptake in both SKBR-3 and MDA-MB-231 cells and (ii), although penetratin uptake was modulated by the treatments in SKBR-3, none of them altered membrane viscosity in this cell line. Membrane compactness or hydration, characterized by the generalized polarization of Laurdan, was correlated weakly and in a statistically non-significant way with penetratin uptake. The predictive value of this correlation is further deteriorated by the fact that the generalized polarization of Laurdan was non-significantly modified by any of the treatments in MDA-MB-231 cells, whereas large and significant effects in penetratin uptake were observed.

In the present experiments, not only did we identify the positive, intramembrane dipole potential inhibiting the uptake and endolysosomal release of penetratin, but we could also enhance the cytoplasmic concentration of the cell penetrating peptide in a medically relevant way by statin treatment. In order for a treatment modality to be of potential medical importance, it must be well-tolerated and therapeutically effective. In contrast to many in vitro studies, in which statins are often overdosed, the nanomolar concentration range of atorvastatin used in the presented experiments is identical to the serum concentration at therapeutic doses (Bjorkhem-Bergman et al., 2011). By applying atorvastatin not requiring metabolic activation, we also circumvented another pitfall of in vitro cellular studies, the application of statins in a prodrug form, which are unlikely to be converted to the active metabolite in cell cultures. Although certain side-effects are associated with statin treatment, extensive experience indicates that they are safe, well-tolerated even in long-term applications in combinations with other drugs (Fievet \& Staels, 2009; Luo et al., 2016). These circumstances further support the potential medical relevance of our findings.

Although reduction of the dipole potential in both MDA-MD-231 and SKBR-3 cells resulted in enhanced penetratin accumulation in the cytosol after phloretin treatment, the latter cell line exhibited lower sensitivity to atorvastatin. Sensitivity to statins correlates inversely with HMG-CoA reductase activity (Göbel et al., 2019; Kimbung et al., 2016). Although the expression of this enzyme is higher by 20\%-30\% in SKBR-3 cells according to a publication (Kimbung et al., 2016) and the Expression Atlas of the European Bioinformatics Institute (https://www.ebi.ac.uk/gxa/home), the confidence intervals of the expression of HMG-CoA reductase in the two cell lines completely overlap according to the Genevestigator platform comparing transcriptomic data from several public repositories, with the level of expression corresponding to the MDA-MB-231 cell line spanning three orders of magnitude. In addition, atorvastatin, albeit at a micromolar concentration, resulted in fourfold induction of HMGCoA reductase expression in SKBR-3 cells, whereas no change in enzyme expression was observed in MDA-MD-231 cells (Kimbung et al., 2016). Cellular uptake of statins is believed to be mediated by transporters to a large extent. Organic anion transporter polypeptides (OATP) have been implicated in transmembrane import of statins (Dobson \& Kell, 2008). However, none of the OATP transporters is expressed significantly differently according to the previous databases. Therefore, the most likely cause for the different atorvastatin sensitivity of the two cell lines in terms of penetratin uptake and reduction in cellular cholesterol content seems to be the difference in the baseline and statin-induced expression of HMG-CoA reductase expression, but solid conclusions cannot be drawn due to inconsistencies in the literature.

In conclusion, we have shown that a decreased, positive membrane dipole potential significantly increases both the total cellular uptake and endocytic escape of penetratin depending on what kind of treatment is used for modifying the dipole potential. As a result, both medically relevant (atorvastatin) and irrelevant (phloretin) treatments decreasing the dipole potential enhance the concentration of penetratin in the cytoplasm, the compartment most relevant for its therapeutic action. Although the applied treatments do modify other membrane properties, the dipole potential was the strongest predictor of penetratin uptake. These findings are in accordance with the dipole potential-decreasing effect of an $\omega-3$ polyunsaturated fatty acid that correlated with enhanced penetratin uptake (Zákány et al., 2021). The experiments identify the biophysical properties of the membrane in general and the dipole potential in particular, as a potential target for enhancing the delivery of drugs and drug candidates exhibiting low or no transmembrane permeability into cells in animal experiments, human trials or in the clinical setting after further studies clarify the cell type dependence and the in vivo potential of such treatments.

\section{ACKNOWLEDGEMENTS}

This work was supported by research grants from the National Research, Development and Innovation Office, Hungary (K120302, ANN133421, GINOP-2.3.2-15-2016-00020 and GINOP2.3.2-15-2016-00044). The Lendület grant from the Hungarian Academy of Sciences is gratefully acknowledged. This work was completed in the ELTE Thematic Excellence Program supported by the Hungarian Ministry for Innovation and Technology. Project no. 2018-1.2.1-NKP2018-00005 has been implemented with the support provided from the National Research, Development and Innovation Fund of Hungary, financed under the 2018-1.2.1-NKP funding scheme.

\section{AUTHOR CONTRIBUTIONS}

G.B. carried out most of the cellular experimental work. L.K. synthesized penetratin and its fluorescently-labelled derivatives. S.T. and G.F.H. contributed significantly to the flow cytometric experiments. T.K. and F.Z. measured cellular cholesterol content and some of the experiments about the biophysical properties of the 
membrane. G.T. contributed to the experiments exploring the role of endocytosis and lysosomal degradation in penetratin uptake. I.M.M. and P.N. conceived and funded the project. G.B. and L.K. wrote the initial version of the manuscript.

\section{CONFLICT OF INTEREST}

The authors declare no conflict of interest.

\section{DECLARATION OF TRANSPARENCY AND SCIENTIFIC RIGOUR}

This Declaration acknowledges that this paper adheres to the principles for transparent reporting and scientific rigour of preclinical research as stated in the BJP guidelines for Design and Analysis and as recommended by funding agencies, publishers and other organisations engaged with supporting research.

\section{DATA AVAILABILITY STATEMENT}

The data that support the findings of this study are available from the corresponding author upon reasonable request. Some data may not be made available because of privacy or ethical restrictions.

\section{ORCID}

Gyula Batta (D) https://orcid.org/0000-0001-8735-6920

István M. Mándity (D) https://orcid.org/0000-0003-2865-6143

Peter Nagy (D) https://orcid.org/0000-0002-7466-805X

\section{REFERENCES}

Alexander, S. P. H., Kelly, E., Mathie, A., Peters, J. A., Veale, E. L., Armstrong, J. F., Faccenda, E., Harding, S. D., Pawson, A. J., Sharman, J. L., Southan, C., Buneman, O. P., Cidlowski, J. A., Christopoulos, A., Davenport, A. P., Fabbro, D., Spedding, M., Striessnig, J., Davies, J. A., ... CGTP Collaborators. (2019). The Concise Guide to PHARMACOLOGY 2019/20: Introduction and other protein targets. British Journal of Pharmacology, 176, S1-S20. https://doi.org/ 10.1111/bph.14747

Bjorkhem-Bergman, L., Lindh, J. D., \& Bergman, P. (2011). What is a relevant statin concentration in cell experiments claiming pleiotropic effects? British Journal of Clinical Pharmacology, 72, 164-165. https:// doi.org/10.1111/j.1365-2125.2011.03907.x

Burlina, F., Sagan, S., Bolbach, G., \& Chassaing, G. (2005). Quantification of the cellular uptake of cell-penetrating peptides by MALDI-TOF mass spectrometry. Angewandte Chemie (International Ed. in English), 44, 4244-4247. https://doi.org/10.1002/anie.200500477

Christensen, M. V., Kongstad, K. T., Sondergaard, T. E., Staerk, D., Nielsen, H. M., Franzyk, H., \& Wimmer, R. (2019). ${ }^{19}$ F-substituted amino acids as an alternative to fluorophore labels: Monitoring of degradation and cellular uptake of analogues of penetratin by ${ }^{19} \mathrm{~F} \mathrm{NMR}$. Journal of Biomolecular NMR, 73, 167-182. https://doi.org/10.1007/ s10858-019-00239-3

Cladera, J., \& O'Shea, P. (1998). Intramembrane molecular dipoles affect the membrane insertion and folding of a model amphiphilic peptide. Biophysical Journal, 74, 2434-2442. https://doi.org/10.1016/S00063495(98)77951-2

Clarke, R. J. (2015). Dipole-potential-mediated effects on ion pump kinetics. Biophysical Journal, 109, 1513-1520. https://doi.org/10.1016/j. bpj.2015.08.022

Clarke, R. J., \& Kane, D. J. (1997). Optical detection of membrane dipole potential: Avoidance of fluidity and dye-induced effects. Biochimica et
Biophysica Acta, 1323, 223-239. https://doi.org/10.1016/S00052736(96)00188-5

Corsini, A., Maggi, F. M., \& Catapano, A. L. (1995). Pharmacology of competitive inhibitors of HMG-CoA reductase. Pharmacological Research, 31, 9-27. https://doi.org/10.1016/1043-6618(95)80042-5

Crismaru, I., Pantea Stoian, A., Bratu, O. G., Gaman, M. A., Stanescu, A. M. A., Bacalbasa, N., \& Diaconu, C. C. (2020). Low-density lipoprotein cholesterol lowering treatment: The current approach. Lipids in Health and Disease, 19, 85. https://doi.org/10.1186/s12944020-01275-x

Curtis, M. J., Alexander, S., Cirino, G., Docherty, J. R., George, G. H., Giembycz, M. A., Hoyer, D., Insel, P. A., Izzo, A. A., Ji, Y., MacEwan, D. J., Sobey, C. G., Stanford, S. C., Teixeira, M. M., Wonnacott, S., \& Ahluwali, A. (2018). Experimental design and analysis and their reporting II: updated and simplified guidance for authors and peer reviewers. British Journal of Pharmacology, 175, 987-993. https:// doi.org/10.1111/bph.14153

Dawaliby, R., Trubbia, C., Delporte, C., Noyon, C., Ruysschaert, J. M., van Antwerpen, P., \& Govaerts, C. (2016). Phosphatidylethanolamine is a key regulator of membrane fluidity in eukaryotic cells. The Journal of Biological Chemistry, 291(7), 3658-3667. https://doi.org/10.1074/jbc. M115.706523

Derossi, D., Joliot, A. H., Chassaing, G., \& Prochiantz, A. (1994). The third helix of the Antennapedia homeodomain translocates through biological membranes. The Journal of Biological Chemistry, 269, 10444-10450. https://doi.org/10.1016/S0021-9258(17)34080-2

Dobson, P. D., \& Kell, D. B. (2008). Carrier-mediated cellular uptake of pharmaceutical drugs: An exception or the rule? Nature Reviews. Drug Discovery, 7, 205-220. https://doi.org/10.1038/nrd2438

Endo, A., Tsujita, Y., Kuroda, M., \& Tanzawa, K. (1977). Inhibition of cholesterol synthesis in vitro and in vivo by ML-236A and ML-236B, competitive inhibitors of 3-hydroxy-3-methylglutaryl-coenzyme $A$ reductase. European Journal of Biochemistry, 77, 31-36. https://doi. org/10.1111/j.1432-1033.1977.tb11637.x

Erazo-Oliveras, A., Muthukrishnan, N., Baker, R., Wang, T. Y., \& Pellois, J. P. (2012). Improving the endosomal escape of cellpenetrating peptides and their cargos: Strategies and challenges. Pharmaceuticals (Basel), 5, 1177-1209. https://doi.org/10.3390/ ph5111177

Fievet, C., \& Staels, B. (2009). Combination therapy of statins and fibrates in the management of cardiovascular risk. Current Opinion in Lipidology, 20, 505-511. https://doi.org/10.1097/MOL.0b013e328332e9ef

Fischer, R., Hufnagel, H., \& Brock, R. (2010). A doubly labeled penetratin analogue as a ratiometric sensor for intracellular proteolytic stability. Bioconjugate Chemistry, 21, 64-73. https://doi.org/10.1021/ bc900301k

Flewelling, R. F., \& Hubbell, W. L. (1986). The membrane dipole potential in a total membrane potential model. Applications to hydrophobic ion interactions with membranes. Biophysical Journal, 49, 541-552. https://doi.org/10.1016/S0006-3495(86)83664-5

Fominaya, J., Bravo, J., \& Rebollo, A. (2015). Strategies to stabilize cell penetrating peptides for in vivo applications. Therapeutic Delivery, 6, 1171-1194. https://doi.org/10.4155/tde.15.51

Futaki, S. (2006). Oligoarginine vectors for intracellular delivery: Design and cellular-uptake mechanisms. Biopolymers, 84, 241-249. https:// doi.org/10.1002/bip.20421

Göbel, A., Breining, D., Rauner, M., Hofbauer, L. C., \& Rachner, T. D. (2019). Induction of 3-hydroxy-3-methylglutaryl-CoA reductase mediates statin resistance in breast cancer cells. Cell Death \& Disease, 10, 91. https://doi.org/10.1038/s41419-019-1322-x

Gross, E., Bedlack, R. S. Jr., \& Loew, L. M. (1994). Dual-wavelength ratiometric fluorescence measurement of the membrane dipole potential. Biophysical Journal, 67, 208-216. https://doi.org/10.1016/ S0006-3495(94)80471-0 
Guidotti, G., Brambilla, L., \& Rossi, D. (2017). Cell-penetrating peptides: From basic research to clinics. Trends in Pharmacological Sciences, 38, 406-424. https://doi.org/10.1016/j.tips.2017.01.003

Jiang, T., Olson, E. S., Nguyen, Q. T., Roy, M., Jennings, P. A., \& Tsien, R. Y. (2004). Tumor imaging by means of proteolytic activation of cell-penetrating peptides. Proceedings of the National Academy of Sciences of the United States of America, 101, 17867-17872. https:// doi.org/10.1073/pnas.0408191101

Joliot, A., Pernelle, C., Deagostini-Bazin, H., \& Prochiantz, A. (1991). Antennapedia homeobox peptide regulates neural morphogenesis. Proceedings of the National Academy of Sciences of the United States of America, 88, 1864-1868. https://doi.org/10.1073/pnas.88.5. 1864

Jones, P., Kafonek, S., Laurora, I., \& Hunninghake, D. (1998). Comparative dose efficacy study of atorvastatin versus simvastatin, pravastatin, lovastatin, and fluvastatin in patients with hypercholesterolemia (the CURVES study). The American Journal of Cardiology, 81, 582-587. https://doi.org/10.1016/S0002-9149(97)00965-X

Kimbung, S., Lettiero, B., Feldt, M., Bosch, A., \& Borgquist, S. (2016). High expression of cholesterol biosynthesis genes is associated with resistance to statin treatment and inferior survival in breast cancer. Oncotarget, 7, 59640-59651. https://doi.org/10.18632/oncotarget. 10746

Kovács, T., Batta, G., Hajdu, T., Szabó, A., Váradi, T., Zákány, F., Csomós, I., Szöllösi, J., \& Nagy, P. (2016). The dipole potential modifies the clustering and ligand binding affinity of ErbB proteins and their signaling efficiency. Scientific Reports, 6, 35850. https://doi.org/10.1038/ srep35850

Kovács, T., Batta, G., Zákány, F., Szöllösi, J., \& Nagy, P. (2017). The dipole potential correlates with lipid raft markers in the plasma membrane of living cells. Journal of Lipid Research, 58, 1681-1691. https://doi.org/ 10.1194/jlr.M077339

Kuhry, J. G., Fonteneau, P., Duportail, G., Maechling, C., \& Laustriat, G. (1983). TMA-DPH: A suitable fluorescence polarization probe for specific plasma membrane fluidity studies in intact living cells. Cell Biophysics, 5, 129-140. https://doi.org/10.1007/BF02796139

Liu, C., Tai, L., Zhang, W., Wei, G., Pan, W., \& Lu, W. (2014). Penetratin, a potentially powerful absorption enhancer for noninvasive intraocular drug delivery. Molecular Pharmaceutics, 11, 1218-1227. https://doi. org/10.1021/mp400681n

Lönn, P., Kacsinta, A. D., Cui, X. S., Hamil, A. S., Kaulich, M., Gogoi, K., \& Dowdy, S. F. (2016). Enhancing endosomal escape for intracellular delivery of macromolecular biologic therapeutics. Scientific Reports, 6 (1), 32301. https://doi.org/10.1038/srep32301

Luo, P., Wang, L., Zhu, H., Du, S., Wang, G., \& Ding, S. (2016). Impact of atorvastatin combined with ezetimibe for the treatment of carotid atherosclerosis in patients with coronary heart disease. Acta Cardiologica Sinica, 32, 578-585. https://doi.org/10.6515/acs20151013h

McCluskey, A., Daniel, J. A., Hadzic, G., Chau, N., Clayton, E. L., Mariana, A., Whiting, A., Gorgani, N. N., Lloyd, J., Quan, A., Moshkanbaryans, L., Krishnan, S., Perera, S., Chircop, M., von Kleist, L., McGeachie, A. B., Howes, M. T., Parton, R. G., Campbell, M., ... Robinson, P. J. (2013). Building a better dynasore: The dyngo compounds potently inhibit dynamin and endocytosis. Traffic, 14 1272-1289. https://doi.org/10.1111/tra.12119

Najjar, K., Erazo-Oliveras, A., Brock, D. J., Wang, T. Y., \& Pellois, J. P. (2017). An L- to D-amino acid conversion in an endosomolytic analog of the cell-penetrating peptide TAT influences proteolytic stability, endocytic uptake, and endosomal escape. The Journal of Biological Chemistry, 292, 847-861. https://doi.org/10.1074/jbc.M116.759837

Nakase, I., Kogure, K., Harashima, H., \& Futaki, S. (2011). Application of a fusiogenic peptide GALA for intracellular delivery. Methods in Molecular Biology, 683, 525-533. https://doi.org/10.1007/978-1-60761919-2_37
O'Shea, P. (2003). Intermolecular interactions with/within cell membranes and the trinity of membrane potentials: Kinetics and imaging. Biochemical Society Transactions, 31, 990-996. https://doi.org/10. 1042/bst0310990

Parasassi, T., De Stasio, G., Ravagnan, G., Rusch, R. M., \& Gratton, E. (1991). Quantitation of lipid phases in phospholipid vesicles by the generalized polarization of Laurdan fluorescence. Biophysical Journal, 60, 179-189. https://doi.org/10.1016/S0006-3495(91)82041-0

Poon, G. M., \& Gariepy, J. (2007). Cell-surface proteoglycans as molecular portals for cationic peptide and polymer entry into cells. Biochemical Society Transactions, 35, 788-793. https://doi.org/10.1042/ BST0350788

Qian, Z., Dougherty, P. G., \& Pei, D. (2015). Monitoring the cytosolic entry of cell-penetrating peptides using a $\mathrm{pH}$-sensitive fluorophore. Chemical Communications, 51, 2162-2165. https://doi.org/10.1039/ C4CC09441G

Qian, Z., Martyna, A., Hard, R. L., Wang, J., Appiah-Kubi, G., Coss, C., Phelps, M. A., Rossman, J. S., \& Pei, D. (2016). Discovery and mechanism of highly efficient cyclic cell-penetrating peptides. Biochemistry, 55, 2601-2612. https://doi.org/10.1021/acs.biochem.6b00226

Repke, H., \& Bienert, M. (1987). Mast cell activation-A receptorindependent mode of substance $P$ action? FEBS Letters, 221, 236-240. https://doi.org/10.1016/0014-5793(87)80932-8

Rezgui, R., Blumer, K., Yeoh-Tan, G., Trexler, A. J., \& Magzoub, M. (2016). Precise quantification of cellular uptake of cell-penetrating peptides using fluorescence-activated cell sorting and fluorescence correlation spectroscopy. Biochimica et Biophysica Acta, 1858, 1499-1506. https://doi.org/10.1016/j.bbamem.2016.03.023

Rothbard, J. B., Jessop, T. C., \& Wender, P. A. (2005). Adaptive translocation: The role of hydrogen bonding and membrane potential in the uptake of guanidinium-rich transporters into cells. Advanced Drug Delivery Reviews, 57, 495-504. https://doi.org/10.1016/j.addr.2004. 10.003

Sarkar, P., Chakraborty, H., \& Chattopadhyay, A. (2017). Differential membrane dipolar orientation induced by acute and chronic cholesterol depletion. Scientific Reports, 7, 4484. https://doi.org/10.1038/ s41598-017-04769-4

Schachter, M. (2005). Chemical, pharmacokinetic and pharmacodynamic properties of statins: An update. Fundamental \& Clinical Pharmacology, 19, 117-125. https://doi.org/10.1111/j.1472-8206.2004.00299.x

Schaefer, E. J., McNamara, J. R., Tayler, T., Daly, J. A., Gleason, J. L., Seman, L. J., Ferrari, A., \& Rubenstein, J. J. (2004). Comparisons of effects of statins (atorvastatin, fluvastatin, lovastatin, pravastatin, and simvastatin) on fasting and postprandial lipoproteins in patients with coronary heart disease versus control subjects. The American Journal of Cardiology, 93(1), 31-39. https://doi.org/10.1016/j.amjcard.2003. 09.008

Sidaway, J. E., Davidson, R. G., McTaggart, F., Orton, T. C., Scott, R. C., Smith, G. J., \& Brunskill, N. J. (2004). Inhibitors of 3-hydroxy-3-methylglutaryl-CoA reductase reduce receptor-mediated endocytosis in opossum kidney cells. Journal of the American Society of Nephrology, 15, 2258-2265. https://doi.org/10.1097/01.ASN. 0000138236.82706.EE

Starke-Peterkovic, T., Turner, N., Vitha, M. F., Waller, M. P., Hibbs, D. E., \& Clarke, R. J. (2006). Cholesterol effect on the dipole potential of lipid membranes. Biophysical Journal, 90, 4060-4070. https://doi.org/10. 1529/biophysj.105.074666

Thoren, P. E., Persson, D., Esbjorner, E. K., Goksor, M., Lincoln, P., \& Norden, B. (2004). Membrane binding and translocation of cellpenetrating peptides. Biochemistry, 43, 3471-3489. https://doi.org/ 10.1021/bi0360049

van den Brand, D., Veelken, C., Massuger, L., \& Brock, R. (2018). Penetration in 3D tumor spheroids and explants: Adding a further dimension to the structure-activity relationship of cell-penetrating 
peptides. Biochimica et Biophysica Acta - Biomembranes, 1860, 1342-1349. https://doi.org/10.1016/j.bbamem.2018.03.007

Via, M. A., Del Popolo, M. G., \& Wilke, N. (2018). Negative dipole potentials and carboxylic polar head groups foster the insertion of cell-penetrating peptides into lipid monolayers. Langmuir, 34, 3102-3111. https://doi.org/10.1021/acs.langmuir.7b04038

Via, M. A., Klug, J., Wilke, N., Mayorga, L. S., \& Del Popolo, M. G. (2018). The interfacial electrostatic potential modulates the insertion of cell-penetrating peptides into lipid bilayers. Physical Chemistry Chemical Physics, 20, 5180-5189. https://doi.org/10.1039/ C7CP07243K

Wallbrecher, R., Depré, L., Verdurmen, W. P., Bovée-Geurts, P. H., van Duinkerken, R. H., Zekveld, M. J., Timmerman, P., \& Brock, R. (2014). Exploration of the design principles of a cell-penetrating bicylic peptide scaffold. Bioconjugate Chemistry, 25(5), 955-964. https://doi. org/10.1021/bc500107f

Wang, F., Wang, Y., Zhang, X., Zhang, W., Guo, S., \& Jin, F. (2014). Recent progress of cell-penetrating peptides as new carriers for intracellular cargo delivery. Journal of Controlled Release, 174, 126-136. https:// doi.org/10.1016/j.jconrel.2013.11.020

Wang, L. (2012). Measurements and implications of the membrane dipole potential. Annual Review of Biochemistry, 81, 615-635. https://doi.org/ 10.1146/annurev-biochem-070110-123033

Xie, J., Bi, Y., Zhang, H., Dong, S., Teng, L., Lee, R. J., \& Yang, Z. (2020). Cell-penetrating peptides in diagnosis and treatment of human diseases: From preclinical research to clinical application. Frontiers in Pharmacology, 11, 697. https://doi.org/10.3389/fphar.2020.00697

Yilmaz, A., Reiss, C., Weng, A., Cicha, I., Stumpf, C., Steinkasserer, A., Daniel, W. G., \& Garlichs, C. D. (2006). Differential effects of statins on relevant functions of human monocyte-derived dendritic cells. Journal of Leukocyte Biology, 79, 529-538. https://doi.org/10.1189/ jlb.0205064

Zákány, F., Kovács, T., Panyi, G., \& Varga, Z. (2020). Direct and indirect cholesterol effects on membrane proteins with special focus on potassium channels. Biochimica et Biophysica Acta - Molecular and Cell Biology of Lipids, 1865, 158706. https://doi.org/10.1016/j.bbalip. 2020.158706
Zákány, F., Pap, P., Papp, F., Kovacs, T., Nagy, P., Peter, M., Szente, L., Panyi, G., \& Varga, Z. (2019). Determining the target of membrane sterols on voltage-gated potassium channels. Biochimica et Biophysica Acta - Molecular and Cell Biology of Lipids, 1864(3), 312-325. https:// doi.org/10.1016/j.bbalip.2018.12.006

Zákány, F., Szabo, M., Batta, G., Kárpáti, L., Mándity, I. M., Fülöp, P., Varga, Z., Panyi, G., Nagy, P., \& Kovacs, T. (2021). An $\omega-3$, but not an $\omega-6$ polyunsaturated fatty acid decreases membrane dipole potential and stimulates endo-lysosomal escape of penetratin. Frontiers in Cell and Development Biology, 9. https://doi.org/10.3389/fcell.2021. 647300

Zhang, J., Li, Q., Wu, Y., Wang, D., Xu, L., Zhang, Y., Wang, S., Wang, T., Liu, F., Zaky, M. Y., Hou, S., Liu, S., Zou, K., Lei, H., Zou, L., Zhang, Y., \& $\mathrm{Liu}, \mathrm{H}$. (2019). Cholesterol content in cell membrane maintains surface levels of ErbB2 and confers a therapeutic vulnerability in ErbB2-positive breast cancer. Cell Communication and Signaling: CCS, 17, 15. https://doi.org/10.1186/s12964-019-0328-4

Ziegler, A. (2008). Thermodynamic studies and binding mechanisms of cell-penetrating peptides with lipids and glycosaminoglycans. Advanced Drug Delivery Reviews, 60, 580-597. https://doi.org/10. 1016/j.addr.2007.10.005

\section{SUPPORTING INFORMATION}

Additional supporting information may be found online in the Supporting Information section at the end of this article.

How to cite this article: Batta, G., Kárpáti, L., Henrique, G. F., Tóth, G., Tarapcsák, S., Kovacs, T., Zakany, F., Mándity, I. M., \& Nagy, P. (2021). Statin-boosted cellular uptake and endosomal escape of penetratin due to reduced membrane dipole potential. British Journal of Pharmacology, 178(18), 3667-3681. https://doi.org/10.1111/bph.15509 


\section{SUPPLEMENTARY INFORMATION}

Statin-boosted cellular uptake and endosomal escape of penetratin due to reduced membrane dipole potential

Gyula Batta, Levente Kárpáti, Gabriela Fulaneto Henrique, Gabriella Tóth, Szabolcs Tarapcsák, Tamás Kovács, Florina Zákány, István M. Mándity, Peter Nagy 


\section{Supplementary Materials and Methods}

\section{Synthesis of penetratin}

Penetratin (RQIKIWFQNRRMKWKK-amide, molecular weight $2245.75 \mathrm{~g} / \mathrm{mol}$ ) was synthesized manually on TentaGel R RAM (Rapp Polymere, Tübingen, Germany), a low crosslinked polystyrene PEG copolymer resin (the substitution was $0.2 \mathrm{mmol} / \mathrm{g}$ ) by the solid-phase method of Merrifield with standard Fmoc chemistry. All protected amino acids analogues for peptide synthesis were purchased from Iris Biotech (Marktredwitz, Germany). Side chains of Gln and Asn were protected by trityl, those of Trp and Lys with t-butoxycarbonyl, and 2,4,6,7-pentamethyl-2,3dihydrobenzofuran-5-sulfonyl (Pbf) was used for Arg. Amino acids were coupled in a stepwise fashion beginning from the $\mathrm{C}$-terminal. Three equivalents of Fmoc-amino acid together with three equivalents of 2-(7-aza-1H-benzotriazole-1-yl)-1,1,3,3-tetramethyluronium hexafluorophosphate (HATU, Sigma-Aldrich) and six equivalents of diisopropylethylamine (DIPEA, Sigma-Aldrich) were allowed to react for at least 2.5 hours with continuous mixing in dimethylformamide (DMF, SigmaAldrich). Completion of the coupling was assessed by the Kaiser test. Before coupling the next amino acid, the Fmoc group was removed from the $\mathrm{N}$-terminal by treatment with $2 \%$ piperidine, 2\% 1,8-Diazabicyclo[5.4.0] undec-7-ene (DBU) in DMF, followed by six washes with DMF-MeOH$\mathrm{CH}_{2} \mathrm{Cl}_{2}$ (all of them purchased from Sigma-Aldrich). Completion of deprotection was assessed by the Kaiser test.

\section{Fluorescence labeling of penetratin}

After coupling the last, $\mathrm{N}$-terminal amino acid, arginine, its Fmoc protecting group was removed and the chain was elongated by a 6-carbon linker (Fmoc-6-aminohexanoic acid, Iris Biotech). The 6-carbon linker was used for reducing the potential effect of fluorescent dyes on penetratin. The Fmoc group was removed from the N-terminal of the linker. The resin, with the peptide still present, was filtered and divided into two equal parts. Half of penetratin was treated with 1.5 equivalents of AFDye532 N-hydroxysuccinimide ester (molecular weight $723.77 \mathrm{~g} / \mathrm{mol}$, Fluoroprobes, Scottsdale, AZ) and 1.5 equivalents of DIPEA in DMF overnight. The other half of penetratin was reacted with 1.5 equivalents of 5(6)-carboxynaphthofluorescein $N$-succinimidyl ester (molecular weight $573.51 \mathrm{~g} / \mathrm{mol}$, Setareh, Eugene, OR) in the presence of DIPEA in DMF. 
Completion of the coupling was assessed by the Kaiser test. Treatment with 95:2.5:2.5 (v/v) trifluoroacetic acid (TFA)/triisopropylsilane/water and dithiothreitol (all of them from SigmaAldrich) for 3 hours removed the protecting groups from amino acid side chains and released the labeled peptides from the resin. The solutions were filtrated, and the peptides were precipitated with a 15 -fold volume of cold diethyl ether. The crude products were purified by preparative reversed-phase HPLC (JASCO, Victoria, Canada) on a C18 column and lyophilized. The purity of the products (>95\%) was assessed by reversed-phase HPLC (JASCO) equipped with an analytical C18 column. The authenticity of each peptide was confirmed by Bruker electrospray ionization mass spectrometry. The predicted and measured molecular mass of the $(\mathrm{M}+\mathrm{H})^{+}$form of AFDye532penetratin was 2983.59 and 2983.548, respectively, whereas the predicted and measured molecular mass of the $(\mathrm{M}+\mathrm{H})^{+}$variant of NF-penetratin was 2833.33 and 2832.5 , respectively. 


\section{Supplementary figures}
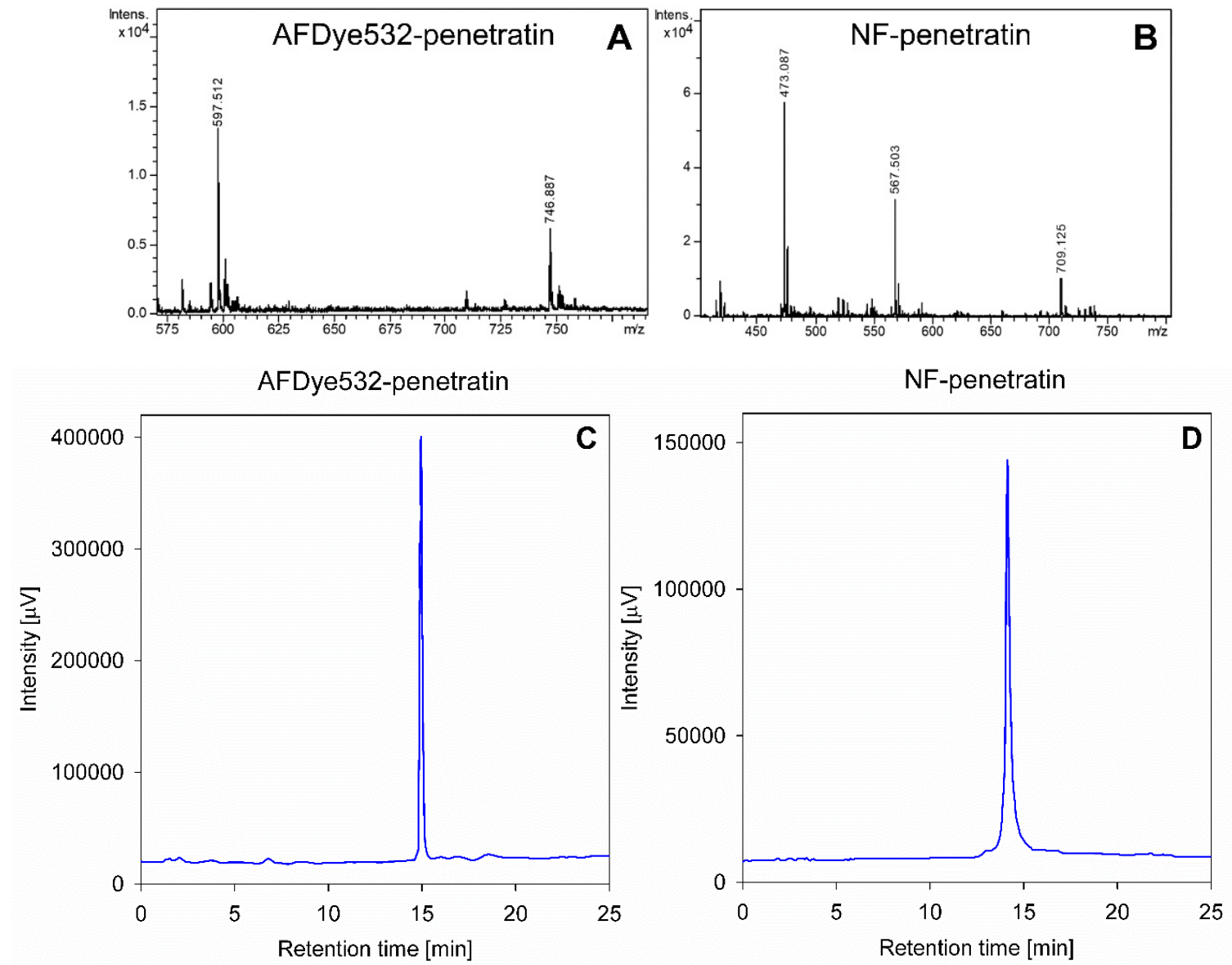

Supplementary Figure 1. Mass spectrometric and HPLC analysis of fluorescently labeled penetratin derivatives. Penetratin labeled with AFDye532 or naphthofluorescein (NF) was analyzed by mass spectrometry $(A, B)$ and HPLC $(C, D)$. Electrospray ionization mass spectrometry was performed with a Bruker micrOTOF instrument using a sample concentration of $0.01 \mathrm{mg} / \mathrm{mL}$ in methanol. The two major peaks in A correspond to the four-times- and five-times-ionized cation of NF-penetratin, whereas the three major peaks in B correspond to cations of AFDye532penetratin with a charge of $+4,+5$ and +6 . Reversed-phase chromatography was performed at a flow rate of $1 \mathrm{~mL} / \mathrm{min}$ using an injected volume of $20 \mu \mathrm{L}$ on an analytical column $(\mathrm{C} 18,250 \times 4.6$ $\mathrm{mm}$, particle size: $5 \mu \mathrm{m}$, pore size: $100 \AA$, Phenomenex Luna, 00G-4252-E0). Elution condition: linear $A B$ gradient where $A$ is $0.1 \%$ TFA/water and $B$ is $80 \%$ acetonitrile/ $0.1 \%$ TFA/20\% water. The fraction of B was increased from $5 \%$ to $80 \%$ in 25 min. Eluted components were quantitated by UV absorption at $220 \mathrm{~nm}$. 


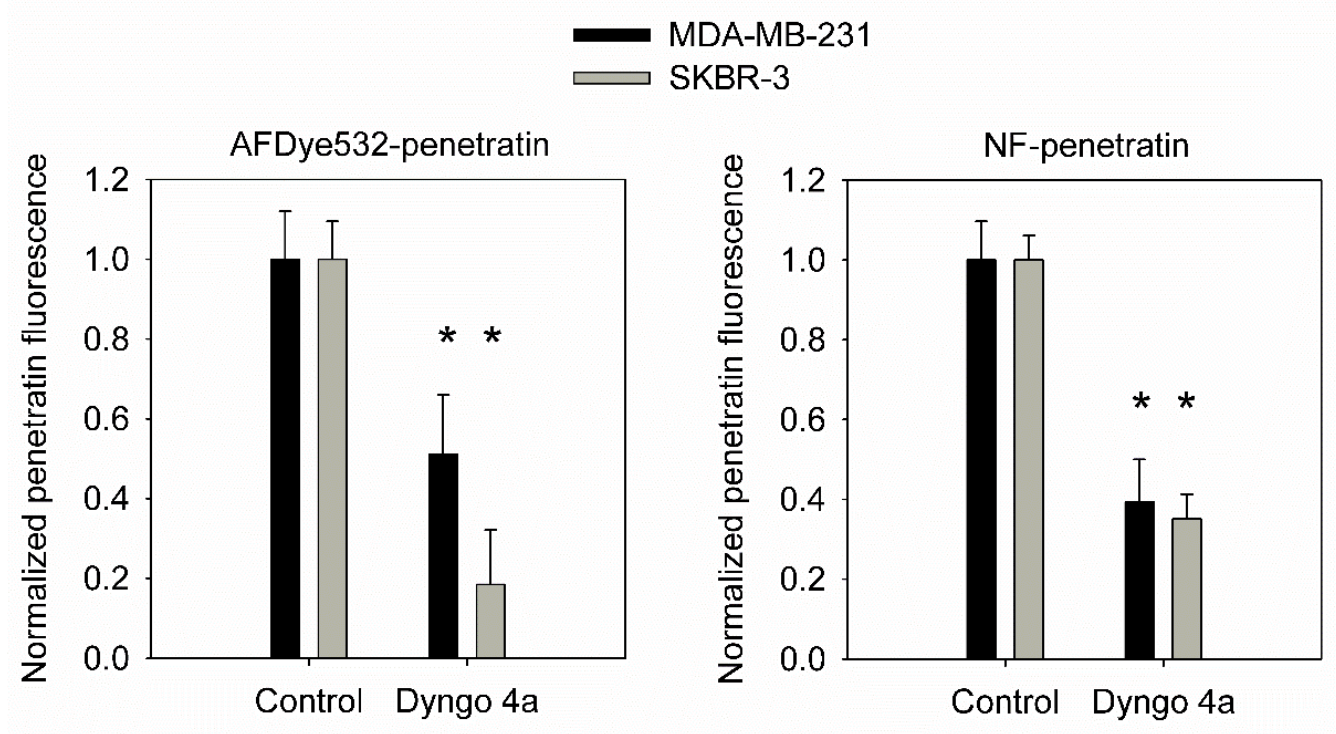

Supplementary Figure 2. Endocytosis plays a significant role in the cellular uptake of penetratin. Cells were pretreated with $40 \mu \mathrm{M}$ Dyngo $4 \mathrm{a}$, an inhibitor of dynamin, followed by incubation with an equimolar mixture of $5 \mu \mathrm{M}$ AFDye532-penetratin and $5 \mu \mathrm{M}$ NF-penetratin. Total cellular penetratin, characterized by the fluorescence of AFDye532-penetratin, and the concentration of penetratin in neutral compartments, characterized by the fluorescence of NF-penetratin, were measured by flow cytometry. The figures show the penetratin fluorescence intensities, normalized to the mean of control samples, at $20 \mathrm{~min}$. The error bars display the standard error of the mean from five biological replicates. Asterisks indicate significant difference compared to the control analyzed by two-way ANOVA followed by Tukey's HSD test $(p<0.05)$. 

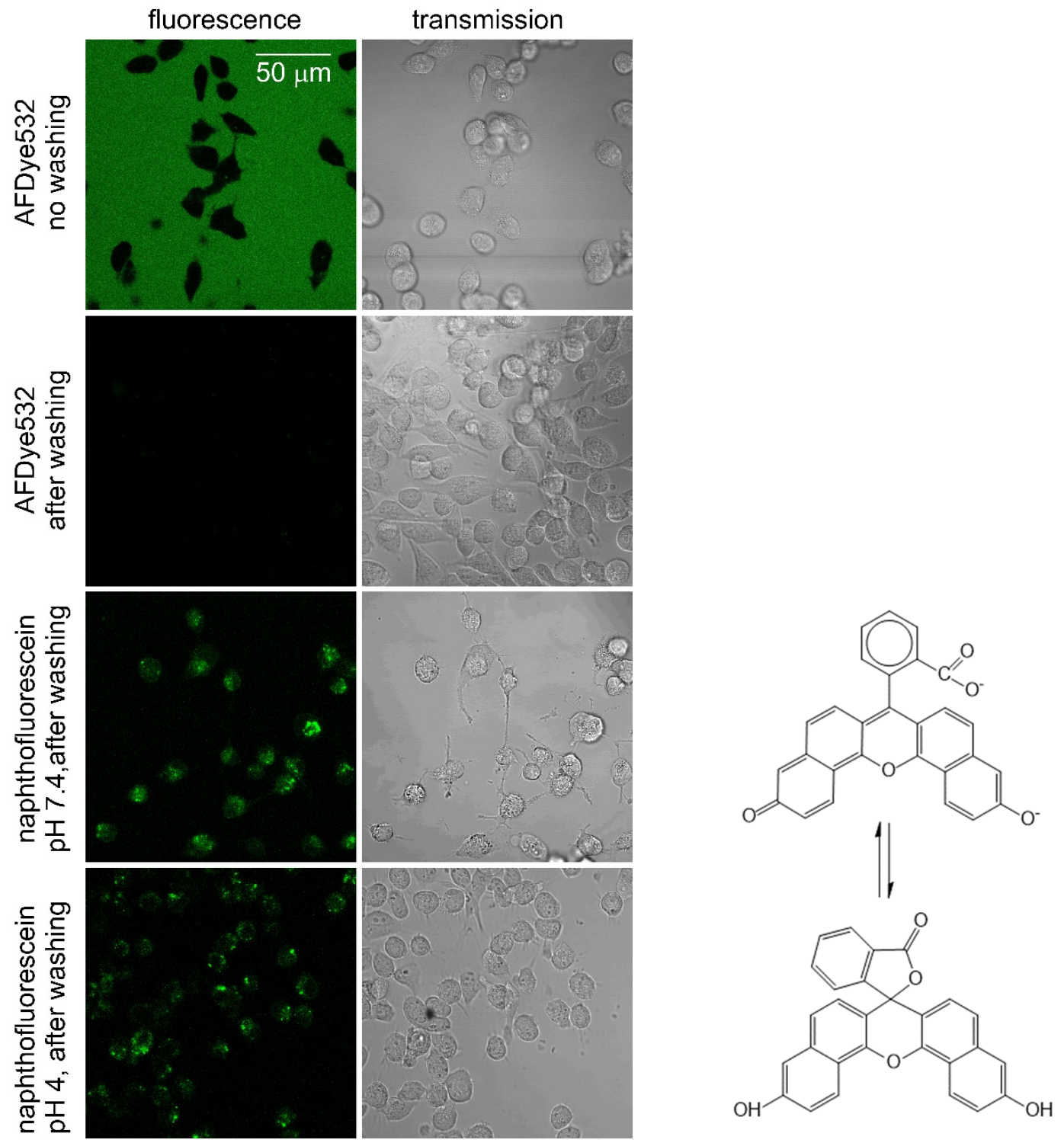

Supplementary Figure 3. Membrane permeability of unconjugated dyes. MDA-MB-231 cells were incubated in the presence of $5 \mu \mathrm{M}$ AFDye 532 or $5 \mu \mathrm{M}$ naphthofluorescein for 20 min at $37^{\circ} \mathrm{C}$ followed by confocal microscopy. AFDye532 images before and after removing the dye from the extracellular space by washing are shown in order to establish that the detection system was capable of recording its fluorescence. Naphthofluorescein undergoes pH-dependent isomerization. Two of the isomers are shown on the right. The dianionic form, present at neutral $\mathrm{pH}$, exhibits the brightest fluorescence (Sjöback, Nygren \& Kubista, 1995), while the neutral form, present at acidic $\mathrm{pH}$, is dark. Uptake of naphthofluorescein was investigated at two different $\mathrm{pH}$ values. Since an acidic extracellular environment may acidify the intracellular space and thereby could quench naphthofluorescein, images were acquired after restoring the extracellular $\mathrm{pH}$ to 7.4. Fluorescence images were contrast-stretched with identical parameters. The scale bar corresponds to every image. Identical results were obtained with SKBR-3 cells (data not shown). 

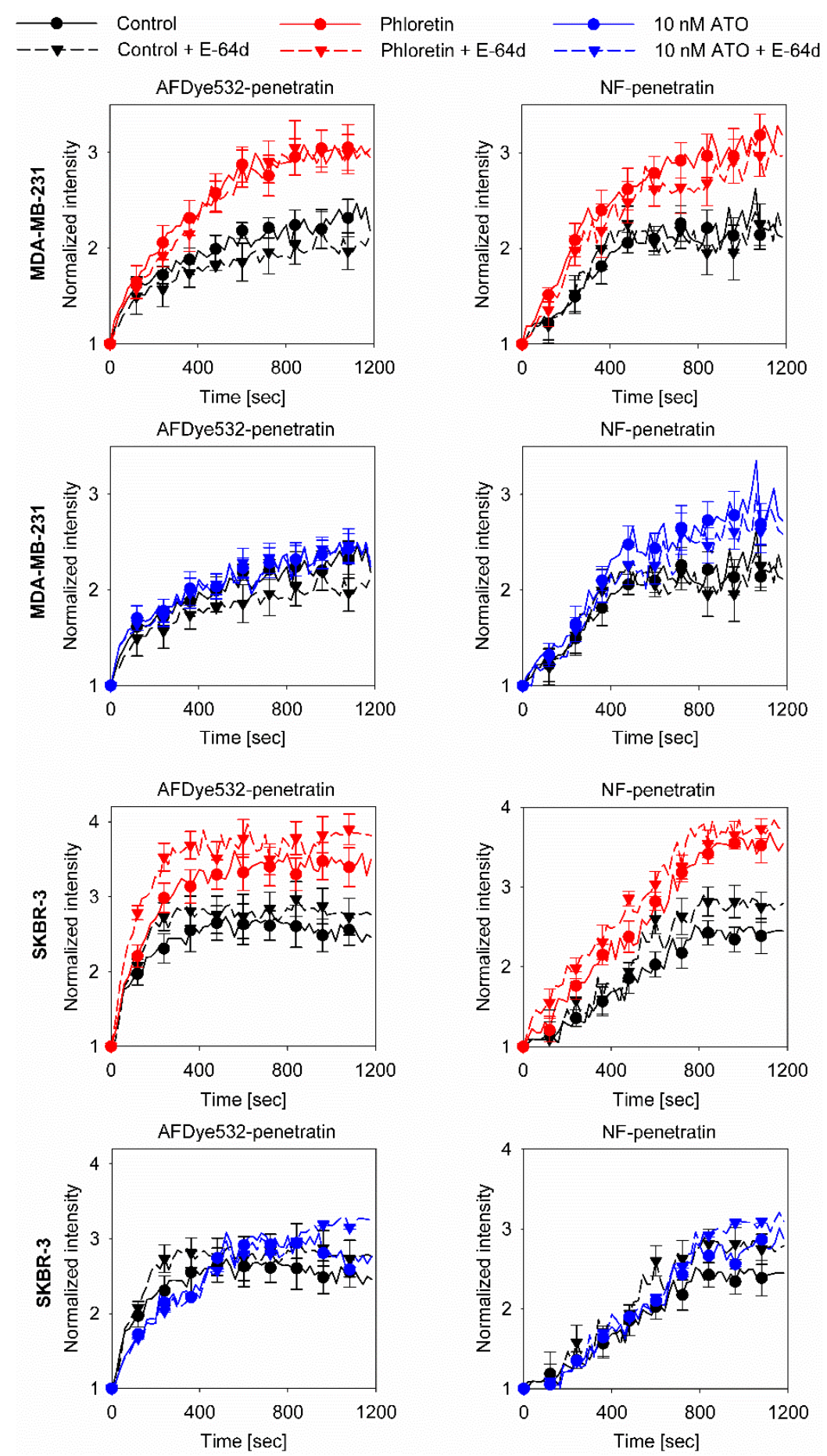

Supplementary Figure 4. Lysosomal degradation of fluorescent penetratin does not modify the fluorescence intensity changes. SKBR-3 and MDA-MB-231 cells were pretreated with the cysteine proteinase inhibitor E-64d followed by carrying out the penetratin uptake experiments in control cells and in those with modified dipole potential as in Figs. 4-5. The error bars represent the standard error of the mean calculated from five biological replicates. The control and E-64dtreated samples are not statistically significantly different from each other ( $p>0.05)$. 


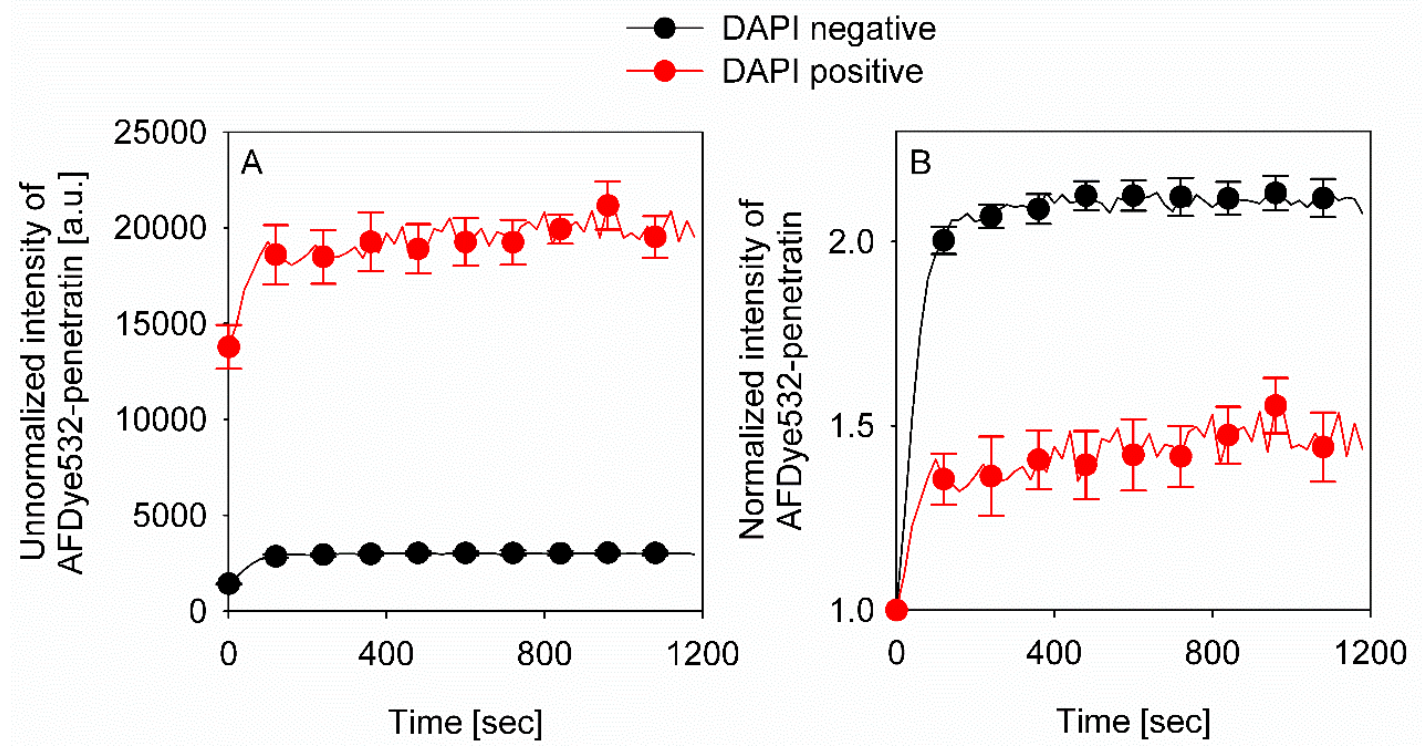

Supplementary Figure 5. Different penetratin uptake of cells with intact and compromised membrane permeability. Cells were incubated in the continuous presence of $5 \mu \mathrm{M}$ AFDye532penetratin and $0.25 \mu \mathrm{g} / \mathrm{mL}$ DAPI at $37^{\circ} \mathrm{C}$, and the fluorescence intensity of cell-associated fluorescent penetratin was measured by time-correlated flow cytometry. Cells were gated on the forward scatter-side scatter dot plot followed by the identification of DAPI-negative and DAPIpositive events corresponding to cells with intact and compromised membrane permeability, respectively. The background-corrected, raw fluorescence intensities ( $A$ ) and those normalized to the initial intensities (B) along with the standard error of the mean determined from five biological replicates are shown in the figure. The error bars on the black symbols in $A$ are smaller than the symbols. The DAPI-positive and DAPI-negative curves are significantly different from each other at each time point (ANOVA followed by Tukey's HSD test, $p<0.05$ ). 


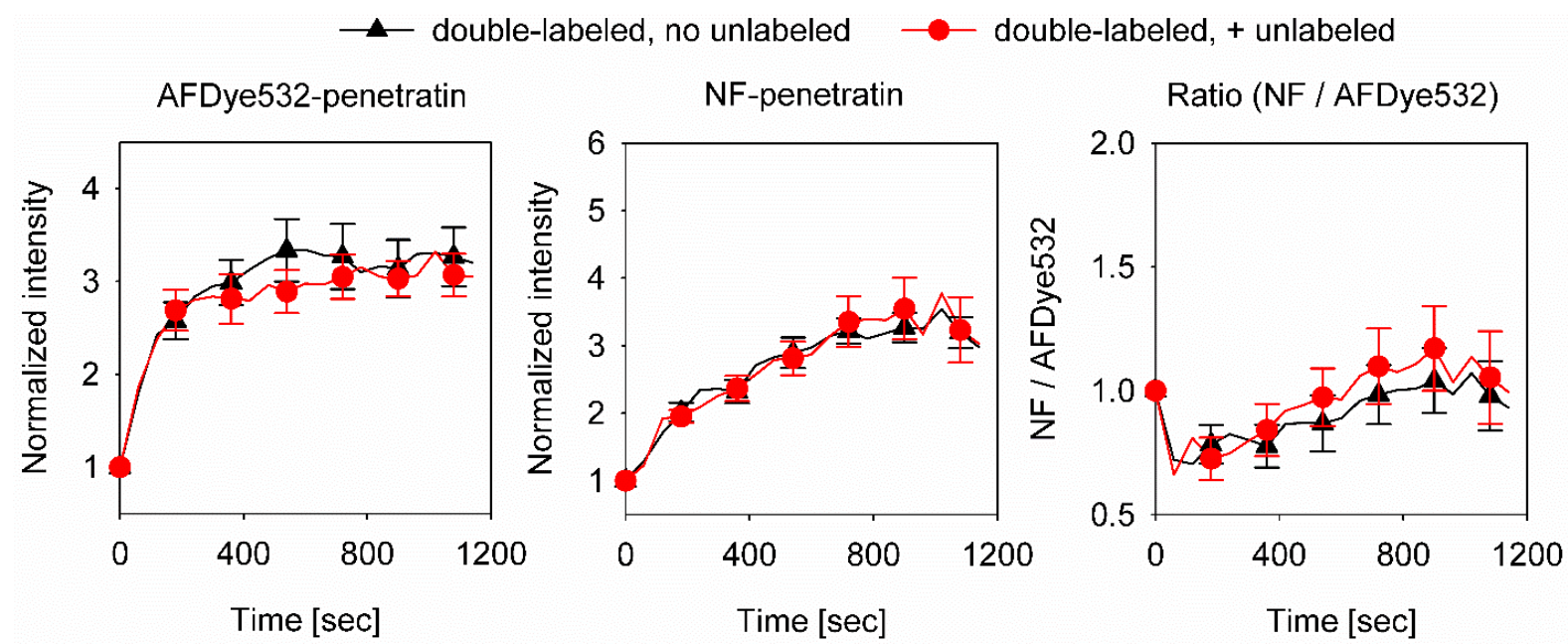

Supplementary Figure 6. Resonance energy transfer between AFDye532 and naphthofluorescein does not influence uptake measurements. Cells were incubated with an equimolar mixture of $5 \mu \mathrm{M}$ AFDye532-penetratin and $5 \mu \mathrm{M}$ NF-penetratin (black) or with this same mixture of the two fluorescent penetratin derivatives supplemented with $10 \mu \mathrm{M}$ unlabeled penetratin (red). Cells were continuously kept in the presence of the peptides at $37^{\circ} \mathrm{C}$, and the fluorescence intensity of cell-associated fluorescent penetratins was measured by time-correlated flow cytometry. Analysis was performed after gating out debris and dead cells. The error bars indicate the standard error of the mean determined from 10 samples from five biological replicates. Statistical comparison of the red and black curves at the final time points did not reveal any significant difference $(p>0.05)$. 


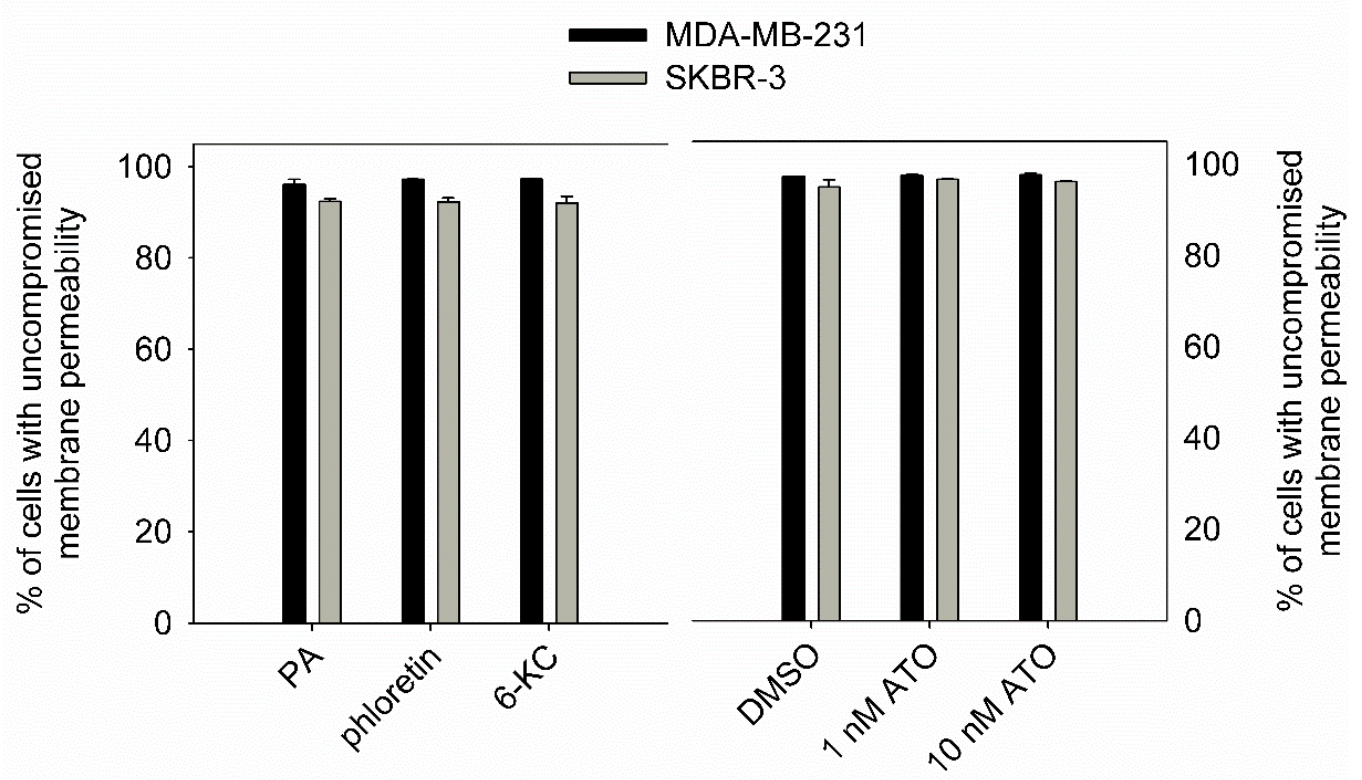

Supplementary Figure 7. The effect of dipole potential-modifying treatments on membrane permeability and cell viability. MDA-MB-231 or SKBR-3 cells were treated with agents altering the dipole potential (6-KC - 6-ketocholestanol; ATO - atorvastatin). Control cells were either treated with Pluronic F-127 (PA) or DMSO. Treatment conditions were identical to those used for measuring penetratin uptake. Cells were then stained with $20 \mu \mathrm{g} / \mathrm{mL}$ propidium iodide (PI) and $8 \mu \mathrm{g} / \mathrm{mL}$ fluorescein-diacetate (FDA) for $5 \mathrm{~min}$ followed by flow cytometry. Viable cells with intact plasma membranes were identified as the PI-negative, FDA-positive subpopulation. The percentage of these cells ( \pm standard error of the mean calculated from five biological replicates) are shown in the figure. 


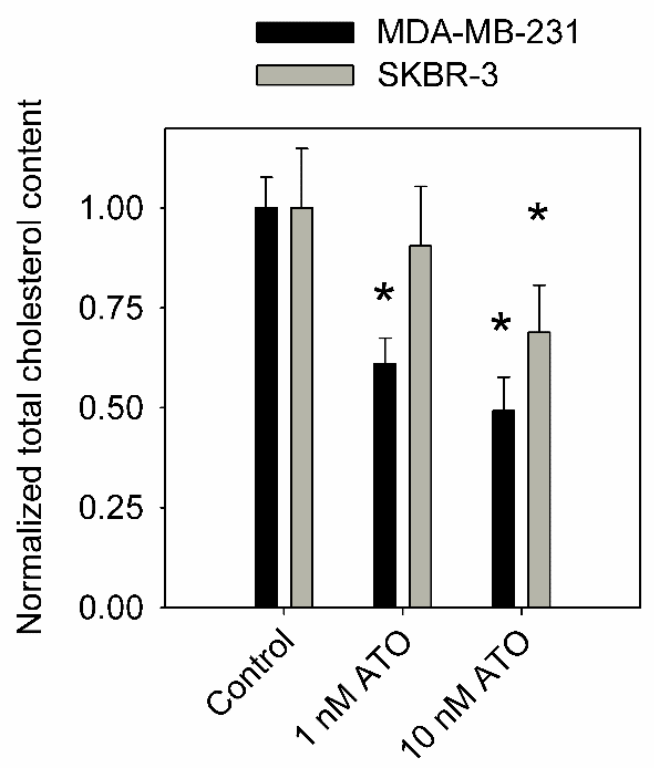

Supplementary Figure 8. The effect of atorvastatin on cellular cholesterol content. MDA-MB-231 and SKBR-3 cells were treated with $1 \mathrm{nM}$ or $10 \mathrm{nM}$ atorvastatin (ATO) for three days. Control cells were treated with DMSO. The total (free + esterified) cholesterol content of cells was determined with Cholesterol Quantitation Kit (Sigma-Aldrich, MAK043). The error bars indicate the standard error of the mean determined from six biological replicates. Statistical significance was tested by analysis of variance followed by Tukey's HSD test. Asterisks indicate significant $(p<0.05)$ difference compared to the control. 


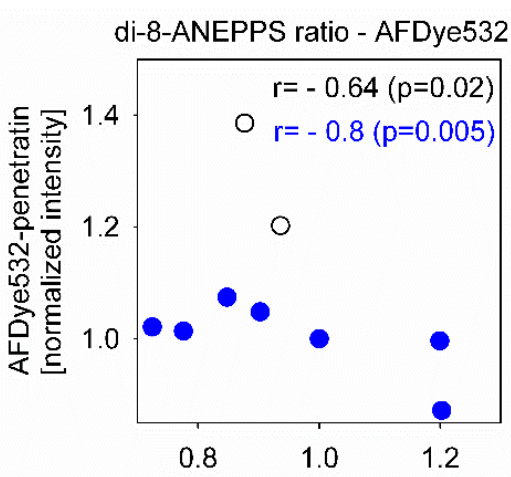

di-8-ANEPPS ratio, normalized
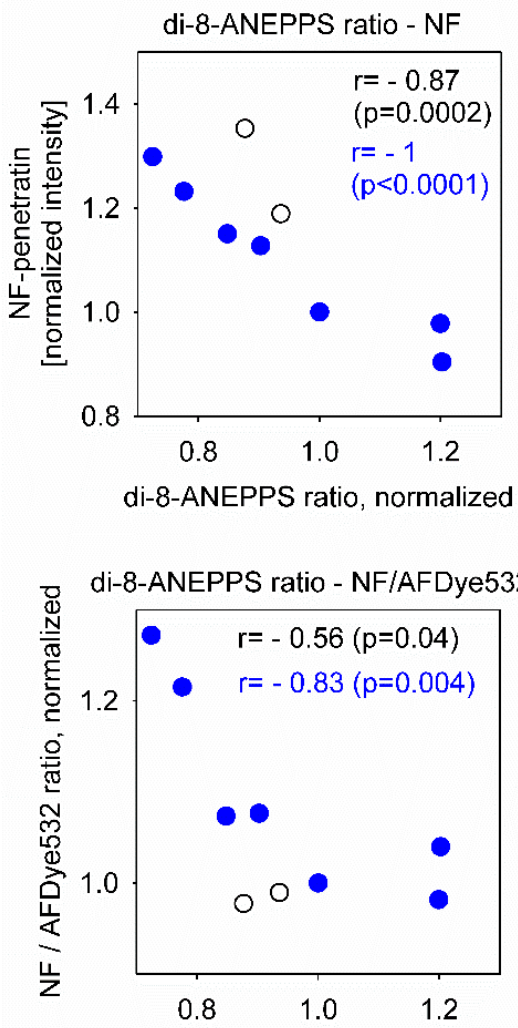

di-8-ANEPPS ratio, normalized
TMA-DPH anisotropy - AFDye532

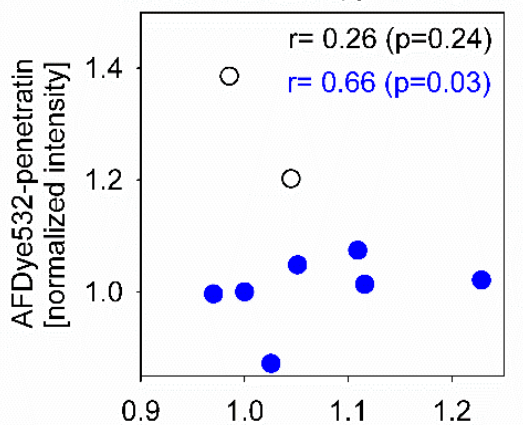

TMA-DPH anisotropy, normalized
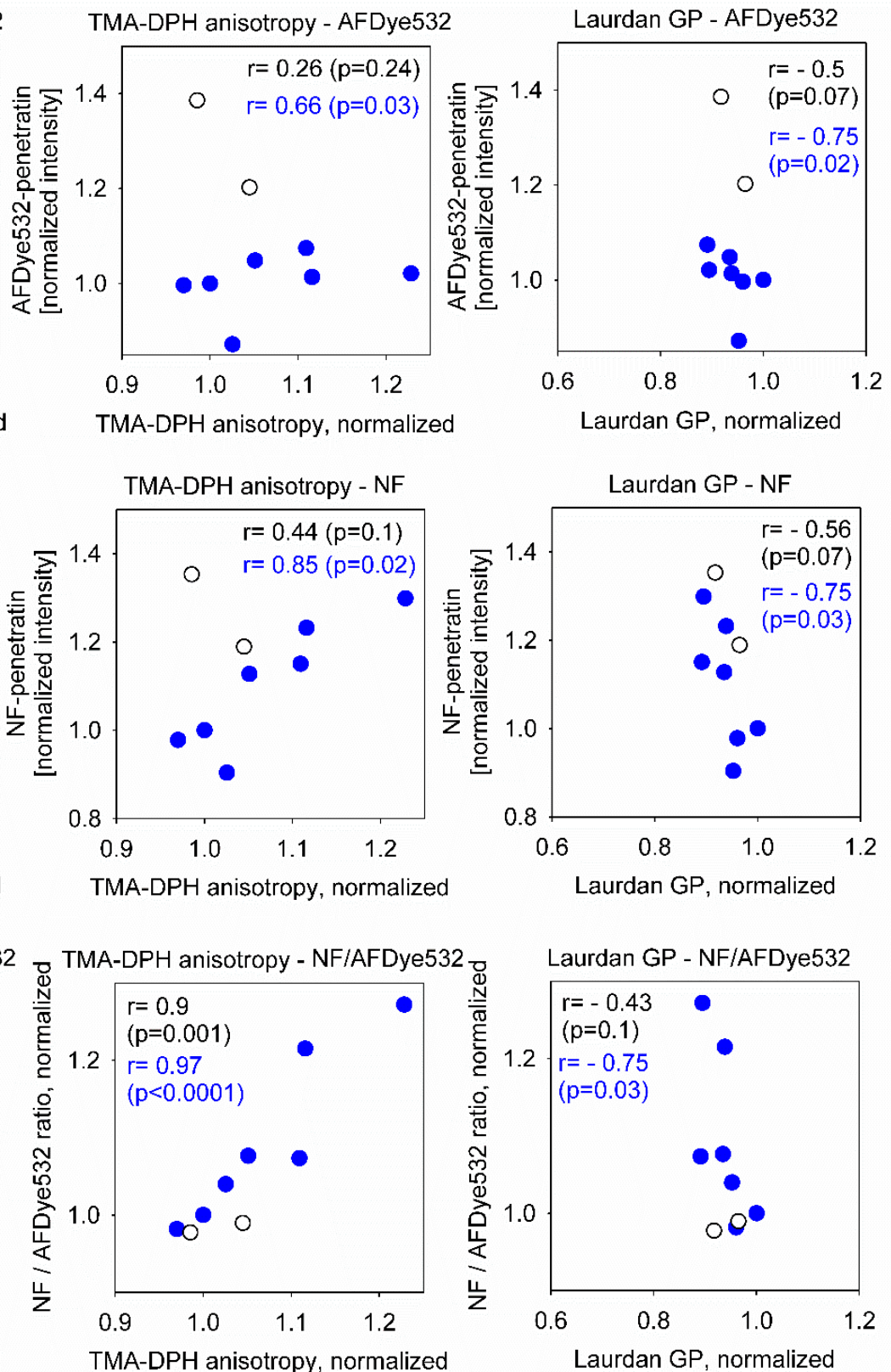

Supplementary Figure 9. Correlation between penetratin uptake and the biophysical properties of the membrane. The dipole potential was measured using di-8-ANEPPS, whose intensity ratio correlates positively with the dipole potential. Membrane fluidity was assessed using the anisotropy of TMA-DPH correlating inversely with fluidity. The generalized polarization (GP) of Laurdan is inversely correlated with hydration and positively correlated with compactness of the membrane. The membrane biophysical properties in these graphs, displayed on the horizontal axes, are identical to those shown in Fig. 3, but they were normalized to the control in the case 
of both cell lines. The vertical axes display normalized penetratin fluorescence intensities. Total penetratin uptake, characterized by AFDye532-penetratin fluorescence at $20 \mathrm{~min}$, was doublenormalized to the initial intensity at $0 \mathrm{~min}$ and to the control sample. Penetratin concentration in the cytoplasm, characterized by NF-penetratin fluorescence at $20 \mathrm{~min}$, was also doublenormalized to the initial intensity at $0 \mathrm{~min}$ and to the control sample. The fractional release of penetratin from acidic compartments is proportional to the NF/AFDye532 intensity ratio. Each point in the figure corresponds to the mean of one particular experimental condition, displayed in Figs. 4 and 5 (regarding penetratin uptake) and Fig. 3 (regarding the membrane biophysical properties). The open, black symbols display the results obtained using phloretin in the two cell lines (MDA-MD-231 and SKBR-3), while the filled, blue symbols correspond to data of all other experimental conditions. The Spearman rank correlation coefficient $(r)$ and its $p$-value for all data points are shown in black, while the blue text displays the same statistical values for the blue data points only, i.e. for all experiments except those with phloretin. 


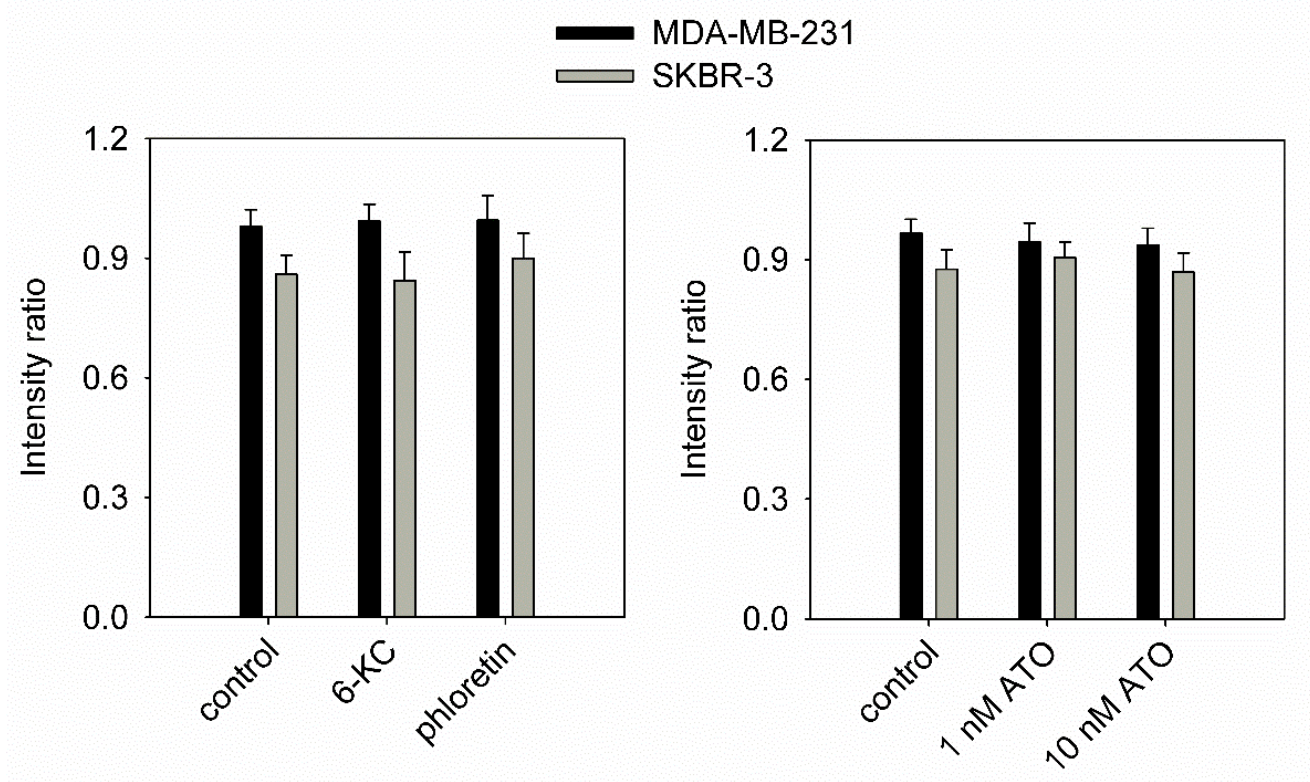

Supplementary Figure 10. The lack of any significant effect of the dipole potential-modifying treatments on lysosomal pH. Cells were treated for modifying the dipole potential as previously, (6-KC - 6-ketocholestanol; ATO - atorvastatin) followed by labeling them with $1 \mu \mathrm{M}$ LysoSensor Yellow/Blue DND-160 (ThermoFisher, L7545) for $10 \mathrm{~min}$. The excitation spectra of the samples were immediately measured by fluorimetry. The emission was measured at $490 \mathrm{~nm}$, and the ratio of intensities excited in the range $305-315 \mathrm{~nm}$ and $360-370 \mathrm{~nm}$ is shown in the figure. This intensity ratio increases as a function of the lysosomal $\mathrm{pH}$ of cells. The error bars represent the standard error of the mean calculated from five biological replicates. Statistical comparison by ANOVA did not reveal significant effects of any of the treatments $(p>0.05)$. The results are presented in two plots since the short-term treatment with 6-kethocholestanol and phloretin and the long-term treatment with atorvastatin were performed separately. 


\section{Supplementary references}

Sjöback R, Nygren J, \& Kubista M (1995). Absorption and fluorescence properties of fluorescein. Spectrochimica Acta Part A: Molecular and Biomolecular Spectroscopy 51: L7-L21. 